\title{
Performance of the Birmingham Solar-Oscillations Network (BiSON)
}

\author{
S.J. Hale ${ }^{1}$ D $~$ R. Howe ${ }^{1}$ • W.J. Chaplin ${ }^{1}$ - G.R. Davies ${ }^{1}$ • \\ Y.P. Elsworth ${ }^{1}$
}

Received: 25 June 2015 / Accepted: 20 October 2015 / Published online: 7 December 2015

(C) The Author(s) 2015. This article is published with open access at Springerlink.com

\begin{abstract}
The Birmingham Solar-Oscillations Network (BiSON) has been operating with a full complement of six stations since 1992. Over 20 years later, we look back on the network history. The meta-data from the sites have been analysed to assess performance in terms of site insolation, with a brief look at the challenges that have been encountered over the years. We explain how the international community can gain easy access to the evergrowing dataset produced by the network, and finally look to the future of the network and the potential impact of nearly 25 years of technology miniaturisation.
\end{abstract}

Keywords Helioseismology, observations · Oscillations, solar

\section{Introduction}

The Birmingham Solar Oscillations Network (BiSON) has now been operating continuously as a six-station network for well over twenty years, recording high-quality spatially unresolved, or "Sun-as-a-star" helioseismic data. It therefore now seems timely to update our previous article on BiSON performance (Chaplin et al., 1996). We present updated results on the temporal coverage and noise performance of the individual sites and the network as a whole and reflect on what we have learned from more than two decades of experience in operating a semi-automated ground-based observing network. These data are available to the wider community through the BiSON Open Data Portal.

The early history of the network has been outlined by Chaplin et al. (1996). In brief, early campaign-style observations from two sites (Haleakala and Izaña) were followed by the addition of an automated observing station in Carnarvon, Western Australia, and later by the deployment of more standardised stations in Sutherland, South Africa, Las Campanas, Chile, and Narrabri, Australia, over the period 1990-1992. The instrument from Haleakala was moved to California and installed in the 60-foot tower at the Mount Wilson Hale Observatory

S.J. Hale

s.j.hale@bham.ac.uk

1 School of Physics and Astronomy, University of Birmingham, Edgbaston, Birmingham B15 2TT, United Kingdom 
in 1992. With occasional instrument upgrades, the network has been operating continuously since then, providing unresolved-Sun helioseismic observations with an average annual duty cycle of about $82 \%$.

\section{Observational Helioseismology: A Brief History}

Oscillations in the velocity field across the Sun were first discovered by Robert Leighton (Leighton, Noyes, and Simon, 1962) using the spectroheliograph developed by George Ellery Hale at the Mount Wilson Hale Observatory in California. The now accepted explanation for these oscillations was developed by Roger Ulrich and John Leibacher, both independently suggesting that sound waves could be generated in the convection zone of the solar interior (Ulrich, 1970; Leibacher and Stein, 1971). Interest in the new field of helioseismology grew quickly, culminating in a dataset six days in length collected from the South Pole (Grec, Fossat, and Pomerantz, 1980), which at the time was the longest and most detailed set of continuous observations available.

It soon became clear that long-term continuous observations were required. The Birmingham group was the first to begin construction of a network of ground-based observatories, beginning with Izaña in Tenerife in 1975 and culminating in six operational sites in 1992.

Other groups have also had success with ground-based networks. Fossat and colleagues went on to deploy the International Research of Interior of the Sun (IRIS; Fossat, 1991) network. The operational strategy of IRIS was different from BiSON, requiring full participation of local scientists responsible for each instrument as opposed to automation. The instrumentation operated in a similar manner to BiSON, observing spatially unresolved global low angular-degree oscillations using an absorption line of sodium, rather than the potassium line used by BiSON. The IRIS network spanned six sites in total (up to nine sites when considering additional collaboration (Salabert et al., 2002a)) and was operational until 2000 (Salabert et al., 2002b; Fossat and IRIS Group, 2002).

Leibacher and colleagues later developed the Global Oscillation Network Group (GONG; Harvey et al., 1996), a six-site automated network using resolved imaging to observe modes of oscillation at medium degree (up to $l \approx 150$ ), complementary to the existing low-degree networks. The sites were chosen in early 1991, and deployment began in 1994 with instruments coming online throughout 1995. The GONG network was upgraded in $2001-2002$ to observe up to around $l=1000$, and is still in operation today.

For completeness, we should also note the LOWL project (Tomczyk, Schou, and Thompson, 1995), which observed at medium degree from one or two sites between 1994 and 2004, and the Taiwanese Oscillations Network (Chou et al., 1995) for high-degree observations, which was deployed between 1993 and 1996, but operated for only a few years.

Several space-based missions have also been successful. In the early 1980s the Active Cavity Radiometer Irradiance Monitor (ACRIM; Willson, 1979) onboard the NASA Solar Maximum Mission spacecraft was sufficiently precise to detect the small changes in intensity caused by the oscillations. Later, the Solar and Heliospheric Observatory (SOHO), a joint project between ESA and NASA, was launched in December 1995 and began normal operations in May 1996 (Domingo, Fleck, and Poland, 1995). Among the suite of instruments onboard the spacecraft are three helioseismic instruments: Global Oscillations at Low Frequencies (GOLF; Gabriel et al., 1995) and Variability of solar Irradiance and Gravity Oscillations (VIRGO; Fröhlich et al., 1995) for low-degree oscillations and Michelson Doppler Imager (MDI; Scherrer et al., 1995) for observations at medium and high degree. The mission was originally planned for just two years, but it is still in operation and currently has a 
mission extension lasting until December 2016, at which point it will have been in service for over twenty years. MDI ceased observations in 2011 when it was superceded by the Helioseismic and Magnetic Imager (HMI; Schou et al., 2012) onboard the Solar Dynamics Observatory (SDO), but GOLF and VIRGO are still in use.

\section{Designing an Automated Robotic Network}

The principle of using resonant scattering spectroscopy to achieve stable and precise measurements of the line-of-sight velocity of the solar atmosphere was first proposed by Isaak (1961). The optical design of the instrument first used at Pic-du-Midi in 1974 to detect long-period solar oscillations is described by Brookes, Isaak, and van der Raay (1976). The basic observational parameter is the Doppler shift of the solar potassium Fraunhofer line at $770 \mathrm{~nm}$. This is achieved through measurement of intensity over a very narrow range in wavelength that sits in the wings of the potassium line so that intensity changes with Doppler shift, and through comparison with the same transition in a potassium vapour in the laboratory, the change in intensity is calibrated to become a measure of velocity. Following initial tests at Pic-du-Midi, the spectrometer was then relocated to the Observatorio del Teide, Tenerife, during 1975. The updated apparatus is described by Brookes, Isaak, and van der Raay (1978a).

The six-station network of today was completed in 1992. There are two stations in each $120^{\circ}$ longitude band, and all of the sites lie at moderate latitudes, around $\pm 30^{\circ}$. The oldest site in the Birmingham Network, Izaña, has now been collecting data for nearly forty years.

The original control system was based around a 40-channel scaler module used for counting pulses from a photomultiplier tube (McLeod, 2002). Timing was controlled by a quartz clock, related to GMT at least once per day, and producing pulses at exactly $1 \mathrm{~s}$ intervals. At the end of each interval two relays would change state and reverse the voltage across an electro-optic modulator, and the data gate would be incremented to the next channel of the scaler. Once all 40 channels of the scaler had been filled, the contents would be destructively written out to magnetic tape, a process that required a further $2 \mathrm{~s}$. The process would then repeat, with each block of data separated by $42 \mathrm{~s}$. The cadence was changed to $40 \mathrm{~s}$ at the beginning of 1990 . This allows for simpler concatenation of data from different sites since there are an integer multiple of $40 \mathrm{~s}$ in a day, and so it provides a network time-standard.

The system was computerised in 1984 using the BBC Microcomputer. The BBC Micro was originally commissioned on behalf of the British Broadcasting Corporation as part of their Computer Literacy Project, and it was designed and built by the Acorn Computer company. Despite the BBC Micro being discontinued in 1994, the spectrometer continued operating in this configuration until 2003, when the computer finally failed and was replaced by a modern PC. A dedicated PIC-based interface was designed to enable the PC to communicate with the original scaler system (Barnes, Jackson, and Miller, 2003, 2004), and this remains the operating configuration today.

For the subsequent fully automated solar observatories, the data-acquisition and control system was initially based around a Hewlett-Packard 3421A data acquisition unit, known colloquially as a "data logger". By the early 1990s these were retired from service and replaced with a standard desktop personal computer and a Keithley System 570 digital input/output interface. The computer ran Microsoft DOS. The limitations of the operating system meant that data could only be retrieved by Birmingham during a scheduled time 
window when the computer would switch from running the data-acquisition program to running a data-transfer program. Over the years a variety of means have been used to return the data from the sites to Birmingham, ranging from tape cartridges or floppy disks sent by post, through direct dial-up modem connections over international phone lines, to the modern internet.

Initially, the instrument at the first automated dome in Carnarvon observed through a glass window. Although the concern regarding site security was low, the window was considered necessary for safe operation. Despite being cleaned regularly by the on-site support, marks on the glass were detrimental to the data, and the decision was eventually made to remove the window and operate in the conventional style of a simple shutter opening. This meant that since the whole system is designed to run completely unattended, careful weather monitoring was required. Both rain and wind sensors are used to close the dome in the event of precipitation or excessive wind. All of the subsequent observatories operated without window glass.

Naturally, repairs have been made over the years and upgrades installed. Probably the most significant is the upgrade to the control software. The original Keithley System 570 data-acquisition system in Carnarvon ran for more than a decade of continuous use, but the units eventually became obsolete as PCs failed and the replacements lacked the required ISA interface. At the same time, the MS-DOS operating system on which the original dome control software relied itself became obsolete, and the Windows software that superseded it for home and office uses was unsuitable for system automation. A new dome control system known as the "Zoo" was developed in the late 1990s (Miller, 2002, 2003) to run under the recently released GNU/Linux operating system (Stallman, 1983; Torvalds, 1991), and this continues in use to the present day.

Much of the old analogue electronics have been gradually replaced with new digital variants. Rather than the whole system consisting of modules in a central rack and communicating through a single interface, the new designs are independent with embedded microcontrollers and communicate with the PC through dedicated RS-232 serial ports. This makes subsequent repairs and upgrades considerably easier since units can be inspected in isolation.

In the following sections we look back over the performance of the network, including temporal coverage and noise levels, and also discuss some of the significant events during the life of the stations.

\section{Data Quality Metrics}

Before we can discuss data quality, we must first define some quality metrics. There are two standard metrics used by BiSON. These are the five-minute figure of merit known simply as the FOM, and the mean high-frequency noise level.

Data from BiSON are collected on a cadence of $40 \mathrm{~s}$, giving an upper limit in the frequency domain (Nyquist frequency) of $12.5 \mathrm{mHz}$. The FOM is a signal-to-noise ratio and is defined as the total power in the main "five-minute" signal band $(2 \mathrm{mHz}-5 \mathrm{mHz})$ divided by the noise $(5.5 \mathrm{mHz}-12.5 \mathrm{mHz})$. When considering the mean noise level in isolation, we look at the mean power in the high-frequency noise $(10.0 \mathrm{mHz}-12.5 \mathrm{mHz})$. We refer to these definitions of FOM and mean noise throughout this article.

Both of these metrics require a certain volume of data in order to ensure that the quality estimates are reliable and that meaningful comparisons can be made between data from 
(a)

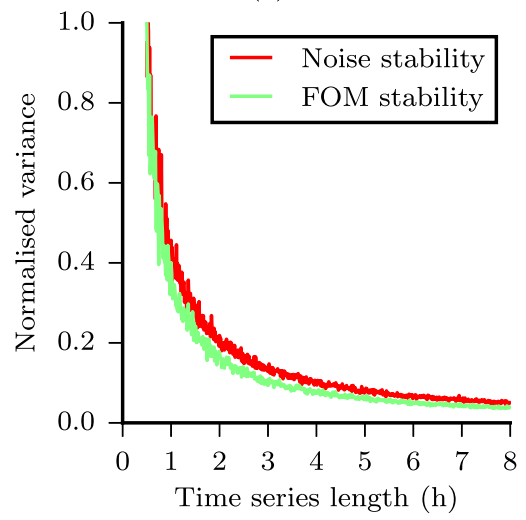

(b)

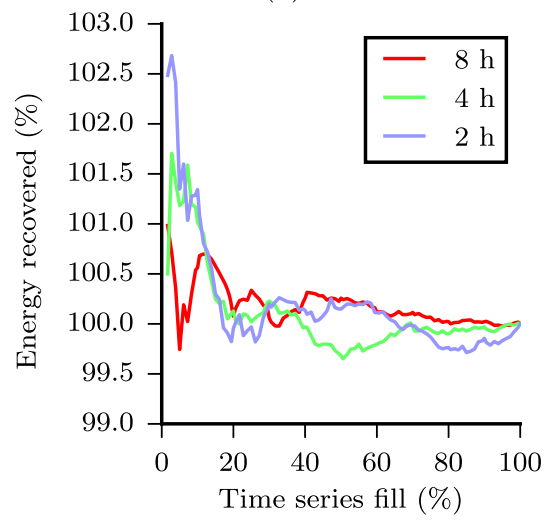

Figure 1 Measured FFT stability over varying dataset length (a) and fill (b). For a valid comparison between datasets from different days and different sites, a time series needs to be at least $3 \mathrm{~h}$ in duration and have a fill of at least $25 \%$.

different days and different instruments. As the length of a dataset decreases, different realisations of random noise begin to have a stronger effect on the estimate of quality. Eventually, the quality estimate becomes so variable as to be meaningless. To determine the minimum dataset length required to make reliable comparisons, a simple artificial dataset was produced with one thousand realisations of random noise. The FOM and mean noise were calculated for each realisation, and the variance in the FOM and mean noise recorded. This was done for dataset lengths varying from thirty minutes up to eight hours. The normalised results are shown in Figure 1(a). Both the quality metrics appear to stabilise at dataset lengths of a minimum of $3 \mathrm{~h}$, and so this was selected as the minimum length that could be reliably used for quality comparison in this article.

An additional problem with data from BiSON is that due to variable weather conditions it is usually not continuous. To be able to compare absolute noise levels, we need to rescale the power spectrum produced by the FFT to compensate for any missing data. This is done by simply dividing by the percentage fill (i.e. the percentage of the total observing time where data are available). To determine how low the fill can be whilst still providing a meaningful estimate of the total energy in an equivalent gap-free dataset, a similar test was used with simple artificial data and one thousand realisations of random noise. A variable size gap was created in three datasets of two, four, and eight hours in total length. The results for the percentage of total energy recovered compared with the original time series are shown in Figure 1(b). Even with very low fills it is possible to recover an estimate of the original total energy to within a few percent. For this article, a fill of at least $25 \%$ was selected as the minimum requirement.

\section{Site Performance}

\subsection{Izaña, Tenerife}

As we saw at the beginning of Section 3, the instrument that was to become the first node of the BiSON was installed at the Observatorio del Teide, Tenerife, in 1975. The Birmingham 
group were the first to establish a global network of ground-based observatories dedicated to helioseismology.

George Isaak, the then head of the High-Resolution Optical Spectroscopy (HiROS) research group, was already considering the development of a permanent network to expand beyond Tenerife long before his seminal article in 1979 on global studies of the five-minute oscillation (Claverie et al., 1979). Campaign-style operations continued throughout 1978 and 1979 at Pic-du-Midi in the French Pyrenees, and a short run at Calar Alto in Spain in 1980. In 1981 the group secured funding to operate a second site on the island of Haleakala in Hawaii, at the Mees Observatory. The instrument at Haleakala operated in much the same way as Mark I in Tenerife. From the two sites together, data were collected for 88 days, producing the longest time series and most highly resolved power spectrum that had been achieved up to that time. However, this was still a long way from year-round complete coverage.

Mark I is housed in the Pyramid Building at the Observatorio del Teide and is run by Pere Pallé and his team of observers (Roca Cortés and Pallé, 2014). Light is collected via two mirrors, known as a cœlostat. The beam is projected through an open window into the apex of the pyramid, where Mark I sits on an optical bench. At the beginning of each observing session, the on-site observer needs to uncover and align the mirrors and start the system. Operator presence is also required throughout the day to close the mirrors in the event of bad weather, and at the end of the observing session.

Despite the higher photon shot noise level compared to our more modern sites, Izaña has been and still is a work-horse of the BiSON network. The site duty-cycle is shown in Figure 2. The duty-cycle is plotted in terms of both number of observational hours and percentage insolation. The insolation compares only the potential daylight hours against actual observational hours and does not differentiate between poor weather conditions and any periods of instrumental failure. Tenerife provides exceptional weather conditions over the summer months, but is rather poor throughout the winter where the conditions become much more variable. There are regular "holes" in the site window function at midday during the spring and autumn months that are caused by cœlostat shadowing. The secondary mirror has two mounting positions, from above or from below, which allows an unobstructed daily run to be achieved throughout the winter and summer months. However, during the change-over period between the two phases, the secondary mirror unavoidably shadows the primary.

The figure of merit (FOM) and mean noise levels for Izaña are shown in Figure 3. The seasonal variation in noise level, and subsequent change in FOM, is due to an effect described by Chaplin et al. $(2004,2005)$. The basic measurement of a BiSON RSS is that of intensity change due to the shift of a solar Fraunhofer line. As the Fraunhofer line shifts due to the line-of-sight relative motion of the solar surface with respect to the laboratory, the measured intensity changes. A data pipeline calibrates the intensity measurements into velocity, taking into account the non-linearity of the Fraunhofer line shape. However, the propagation of noise on the original intensity measurement through the calibration process is non-linear (Hoyng, 1989) due to the varying types of noise. The system encounters white noise, for example due to photon statistics and analogue-to-digital conversion, multiplicative noise due to gain fluctuations, and additive noise due to offset fluctuations, in addition to more random effects due to pointing errors and temperature fluctuations. As the Fraunhofer line shifts daily due to Earth's rotation and seasonally due to the eccentricity of Earth's orbit, the line gradient at the operating point of the instrument changes. This causes the noise level in the derived quantities to change even if the noise level in the basic intensity measurement remains constant. Aside from the seasonal variation, the noise levels from 

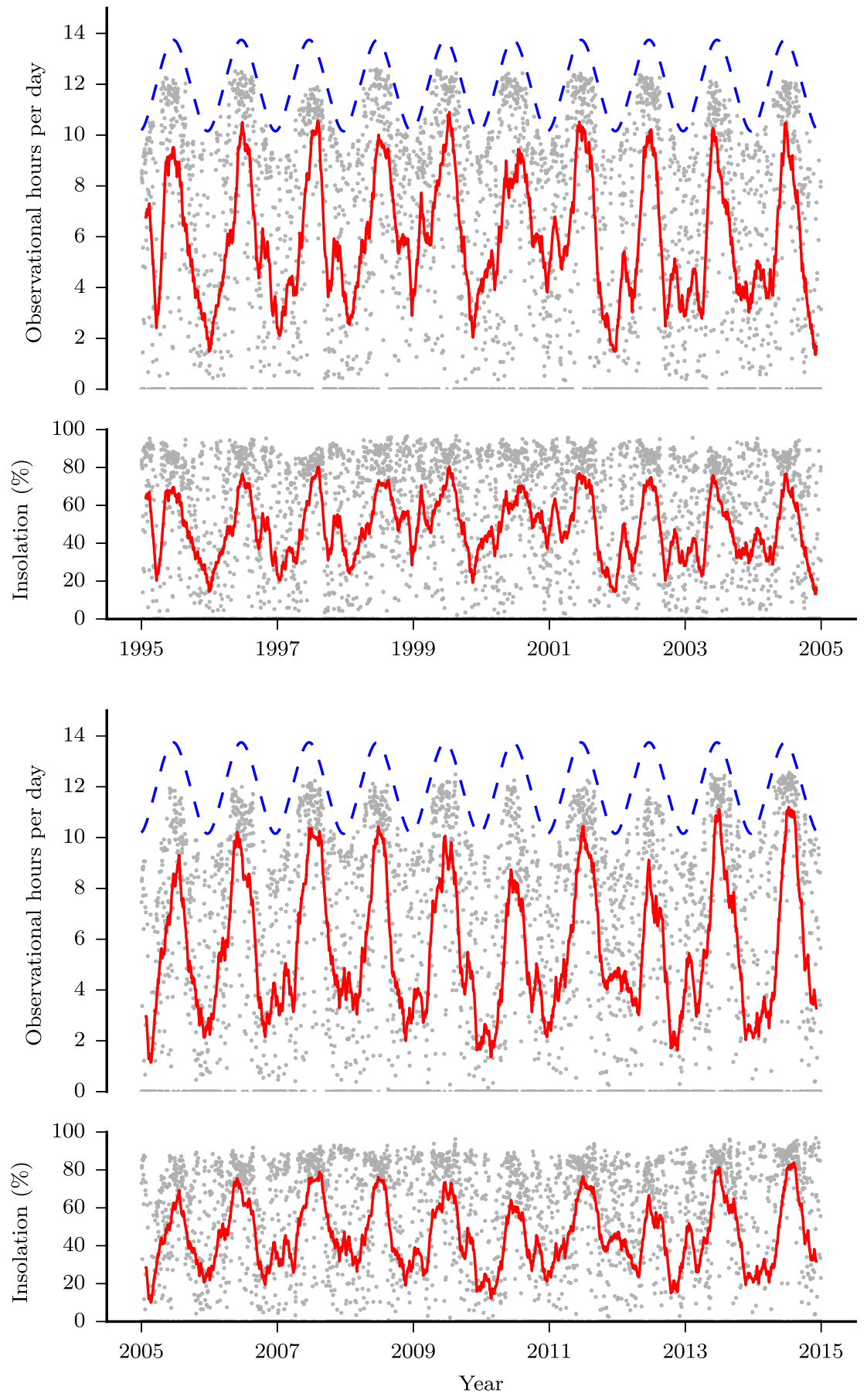

Figure 2 Izaña duty cycle as a function of date, plotted in hours per day, and as a percentage of potential daylight hours. There is one grey dot per day, and the solid red curve represents a 50-day moving mean. The dashed blue curve shows potential daylight hours. 

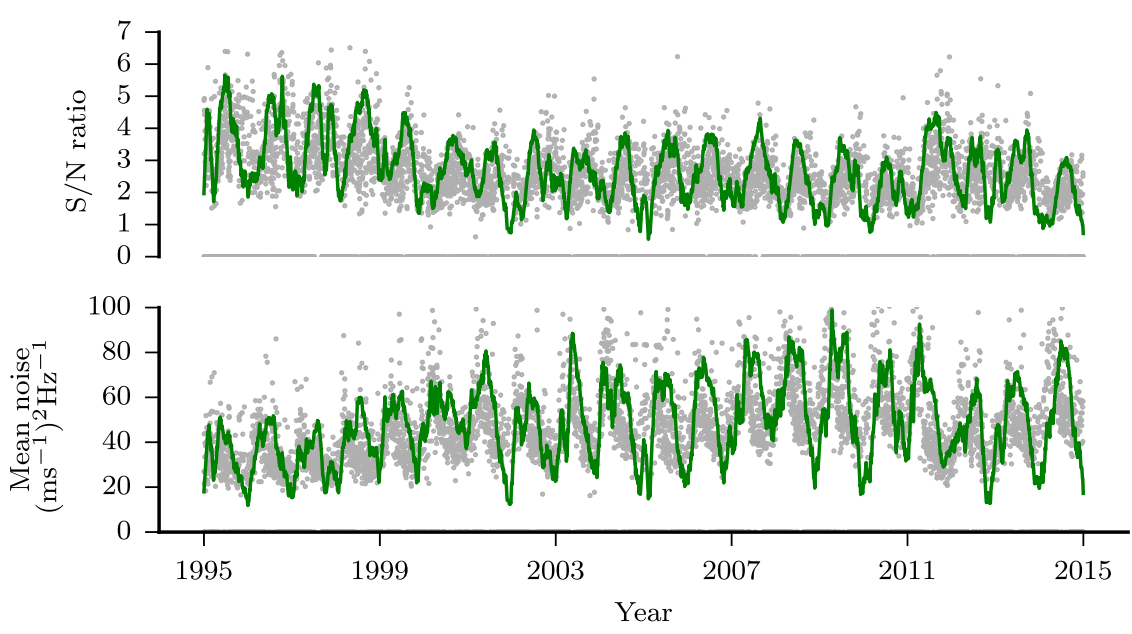

Figure 3 Izaña data quality as a function of date. Top: Signal-to-noise ratio, higher is better. Bottom: Mean noise level, lower is better. There is one grey dot per day, and the solid green curve represents a 50-day moving mean.

Izaña show remarkable stability and offer an unprecedented temporal baseline of almost 40 years.

\subsection{Carnarvon, Western Australia}

When considering expanding to a global network, the group realised that it would not be practical to operate such a network manually. Requiring an observer to be present on site, all day every day for 365 days per year, would be very expensive and potentially unreliable. As the number of network nodes increases, the number of observers required to operate them all year round would become untenable. The key to a reliable network would be automation. For the early 1980s, this was an ambitious endeavour. If successful, it would be one of the first automated astronomical telescopes anywhere in the world, in any field.

From 1981 the group worked on this innovative new design, and by the summer of 1983 , a prototype was ready to test. The new instrument would point directly at the Sun and move on an equatorial mount like a classical astronomical telescope. Moving a mount under computer control would be significantly easier than aligning and pointing mirrors. Testing in Haleakala proved that the system worked, and the group began looking for an installation site. Western Australia was selected as a good longitude to complement coverage from Picdu-Midi and Haleakala. Four sites were investigated: Woomera Rocket Testing Range, Learmonth, Exmouth, and Carnarvon. Isaak decided that Woomera was too dusty, but the other three towns were all suitable. The group settled on Carnarvon, some $900 \mathrm{~km}$ north of Perth. The group arrived in Carnarvon in 1984. Similar testing was carried out to that which had been successful in Haleakala. By the end of the year it was clear that full automation was practical, and the decision was made to push ahead.

Initially, the data collection systems operated on a $42 \mathrm{~s}$ cadence like the existing systems in Izaña and Haleakala. Carnarvon was migrated to the newer 40 s cadence in April 1992. Data obtained previous to this date are interpolated onto the newer standard cadence. Over 
the months following installation, the system worked extremely well. It produced highquality data and proved to be reliable. Most glitches were due to the Australian wildlife - rodents eating through cables, or cockatoos making nests in the upper parts of the dome. The Sun itself caused some problems, with certain types of connector and cable insulation quickly decaying under constant exposure to solar UV. A simple and cheap solution was found for this, which was to wrap exposed cables and components in aluminium cooking foil. The goal of demonstrating that an automated system could work, and work well, had been successfully achieved. Funding to roll out further stations was quickly secured.

Carnarvon has not been without its problems. The PC failed in August 2002, and this is the first drop-out in the Carnarvon duty cycle (Figure 4). In May 2005 a freak rainstorm emptied over three inches of rain in just two hours. This is almost the same amount of rain that Carnarvon expects in a whole year. It was not a good time to find out that the rain detector had failed, and so the dome did not close (Barnes and Hale, 2005; New and Hale, 2006). The whole dome was thoroughly flooded, completely destroying the control electronics for one instrument and severely damaging a second instrument. Water was poured out of some of the electronics. At the time, Carnarvon had two instruments in operation. The primary instrument was able to be repaired on-site after designing new detectors and control electronics in Birmingham. However, the secondary instrument was written off and had to be returned to Birmingham (Barnes, Miller, and Jackson, 2007) for a considerable programme of repairs and upgrades. The new instrument was installed in 2009 (Barnes and Miller, 2009) and provided a substantial improvement in noise level and data quality (Figure 5).

In 2013 the land was bought by NBN Co. Ltd, the National Broadband Network of Australia, as a base for a new satellite internet station. A lease was approved between the group and NBN, and the dome was shut down for just over a month in October 2013 during the NBN construction in an attempt to limit the amount of dust and dirt entering the dome. The NBN compound has two large antennas that are due east of the dome and cause some shadowing in the early morning around the summer solstice. Despite this, the Carnarvon BiSON dome continues to collect data year-round.

\subsection{Sutherland, South Africa}

Following the success of the Carnarvon station, three more sites were commissioned. Some minor changes were made to the original design. The main building became rectangular and made of brick, rather than cylindrical and made from plywood clad with corrugated iron. This substantially increased the floor-area, allowing for more storage space and easier access to the control electronics. The mount was also made considerably larger, allowing for larger and heavier instrumentation.

The first station of this new design was built in Birmingham in 1988. It is located on the roof of the Poynting Physics building. Once the new-style dome had been finalised, the first site to be commissioned was in Sutherland, South Africa, at the South African Astronomical Observatory (SAAO) in 1989 (Davidson and Williams, 2004) and completed in 1990. The observatory itself was established in 1972 and is run by the National Research Foundation of South Africa. It is located in the Karoo Desert, in the Northern Cape of South Africa, approximately $350 \mathrm{~km}$ north-east of Cape Town.

Like Carnarvon, Sutherland started out with a Keithley System 570 data-acquisition system and later got its Zoo upgrade in 2006 (Barnes and Miller, 2006). New digital temperature controllers were installed in 2007 (Barnes, 2008, 2009), new counters in 2012 (Hale, 2012), and a new digital autoguider in 2013 (Hale, 2013).

The Sutherland duty cycle is shown in Figure 6. The drop-out in late 1997 through early 1998 was caused by a range of faults. The tracking motor on the mount failed, and 

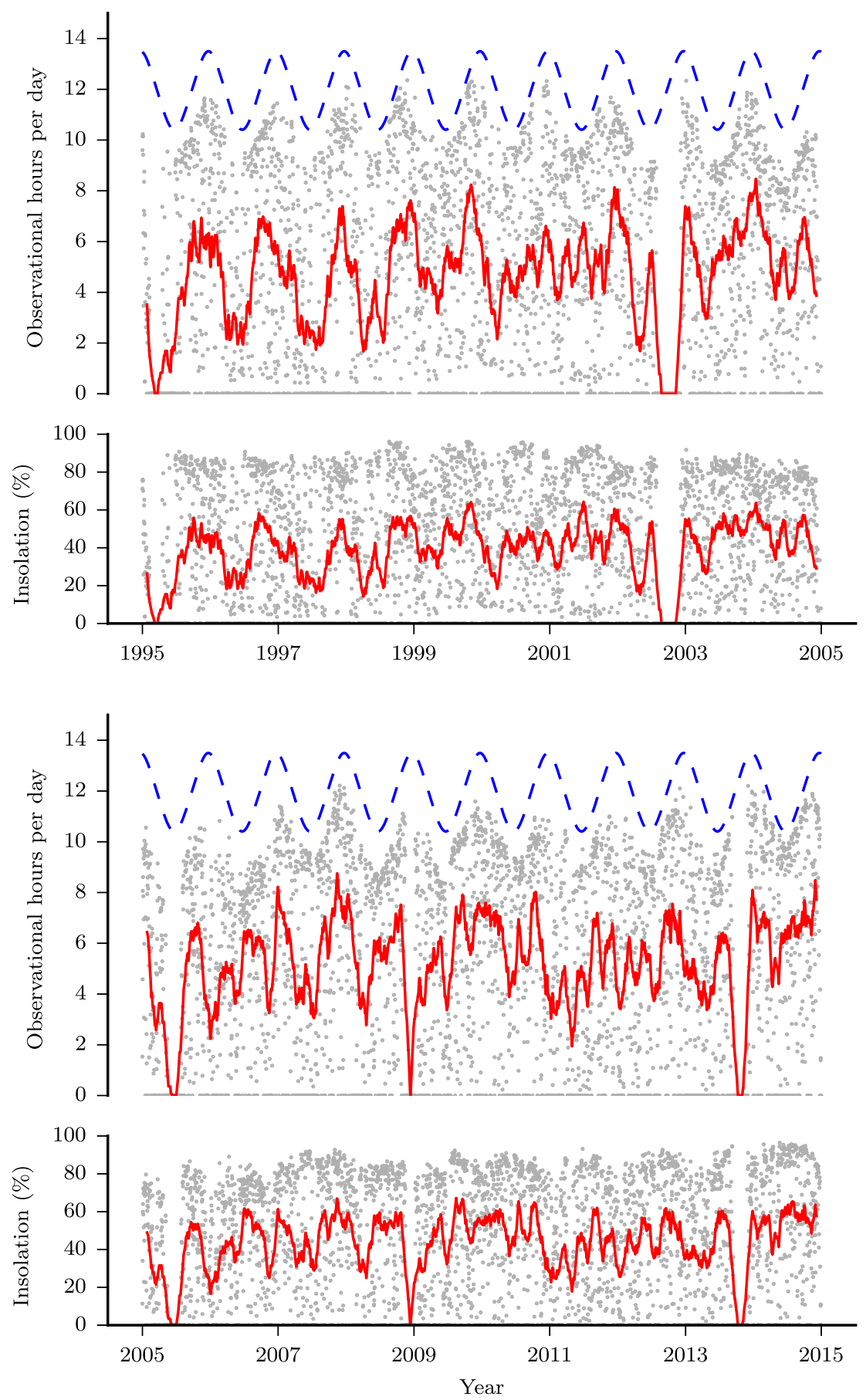

Figure 4 Carnarvon duty cycle as a function of date, plotted in hours per day, and as a percentage of potential daylight hours. There is one grey dot per day, and the solid red curve represents a 50-day moving mean. The dashed blue curve shows potential daylight hours. 

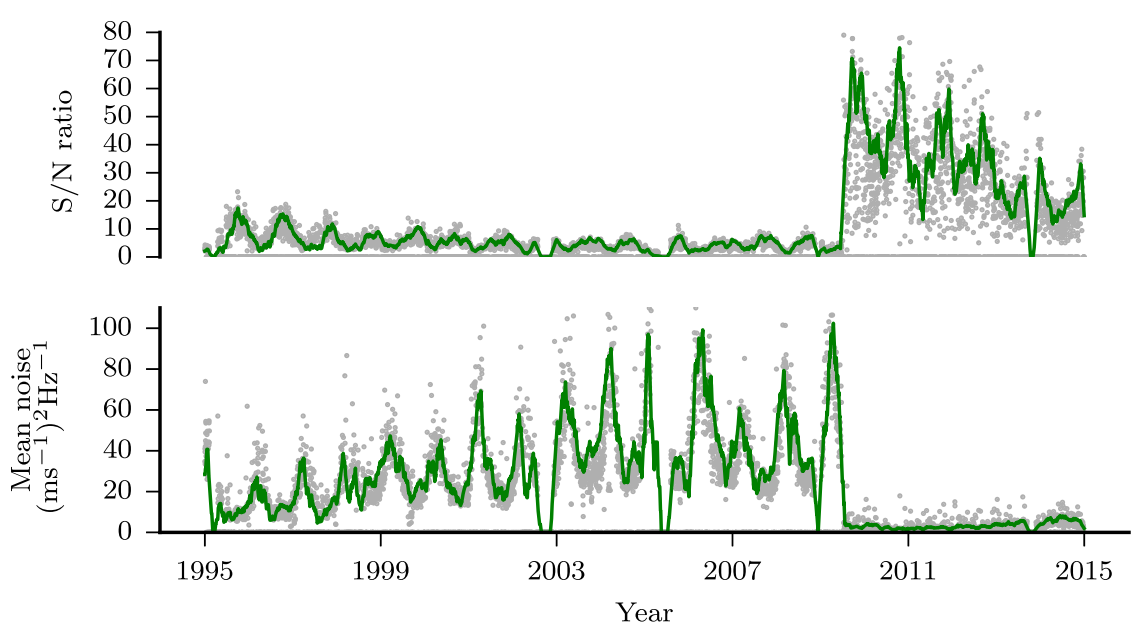

Figure 5 Carnarvon data quality as a function of date. Top: Signal-to-noise ratio, higher is better. Bottom: Mean noise level, lower is better. There is one grey dot per day, and the solid green curve represents a 50-day moving mean. The large step in 2009 is due to installation of a new upgraded spectrometer.

problems with the declination limit switches caused the mount to be unresponsive. Also, the dome moved out of alignment and began shadowing the instrument just before sunset. Later, the electronic polarisation modulator failed, and this was not replaced until late January 1998. In early 1999, a fault developed on the scaler system that counts the pulses produced by the detectors. Following extensive investigation, simply re-seating all the chips on the scaler cards fixed the problem, but not until almost a month of data had been lost. The problem reoccurred several times over the years, until the scaler system was finally replaced completely in 2012. The gap in 2007 was due to some downtime whilst the temperature control systems were upgraded, and in 2008 due to a heavy snow storm.

Looking at the data quality and noise levels (Figure 7), we see that the site has shown consistent performance with gradual improvement as systems were upgraded. The higher noise level in 2000 was due to a sticky declination gearbox. Weather conditions in Sutherland show solid year-round performance.

\subsection{Las Campanas, Chile}

After the completion of Sutherland, a third automated site was opened in Chile in 1991, at the Las Campanas Observatory operated by the Carnegie Institution for Science. Las Campanas Observatory is located in the southern Atacama Desert of Chile, around $100 \mathrm{~km}$ north-east of La Serena. The observatory was established in 1969 and was a replacement for the Mount Wilson Hale Observatory near Pasadena, which had started to experience too much light pollution from the growing city of Los Angeles. The main office is in Las Serena, whilst the headquarters remain in Pasadena.

Las Campanas received its Zoo upgrade in December 2005 (Hale and Miller, 2006), a new digital autoguider in February 2011 (Miller, 2011; Barnes and Miller, 2011), and a new temperature controller in 2015 (Hale, 2015b,c).

The Las Campanas duty cycle is shown in Figure 8. Las Campanas has had a range of problems. One notable experience was in July 1997, when a direct lightning strike to the dome destroyed several pieces of sensitive electronics. Whilst repairs were completed, 

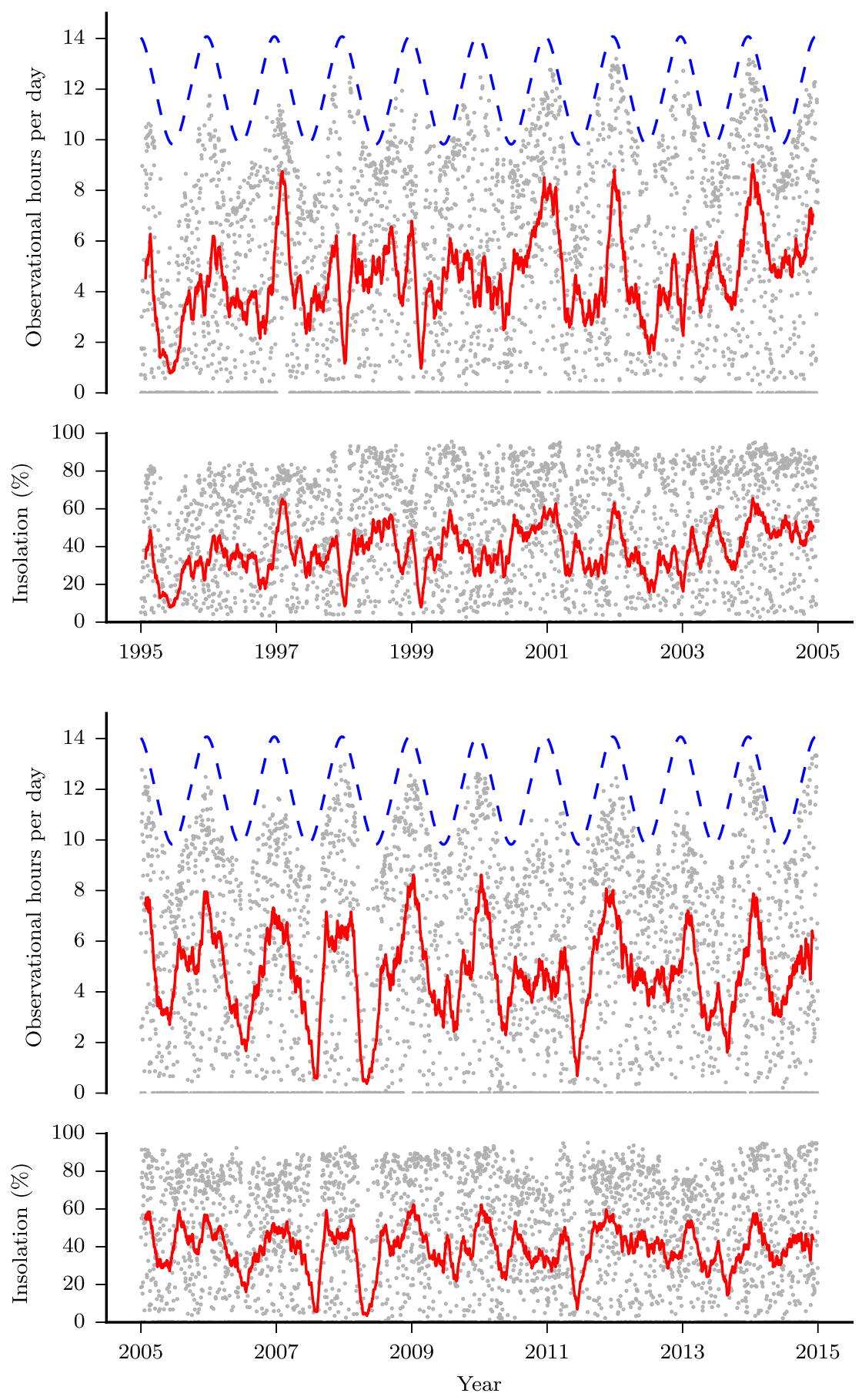

Figure 6 Sutherland duty cycle as a function of date, plotted in hours per day, and as a percentage of potential daylight hours. There is one grey dot per day, and the solid red curve represents a 50-day moving mean. The dashed blue curve shows potential daylight hours. 

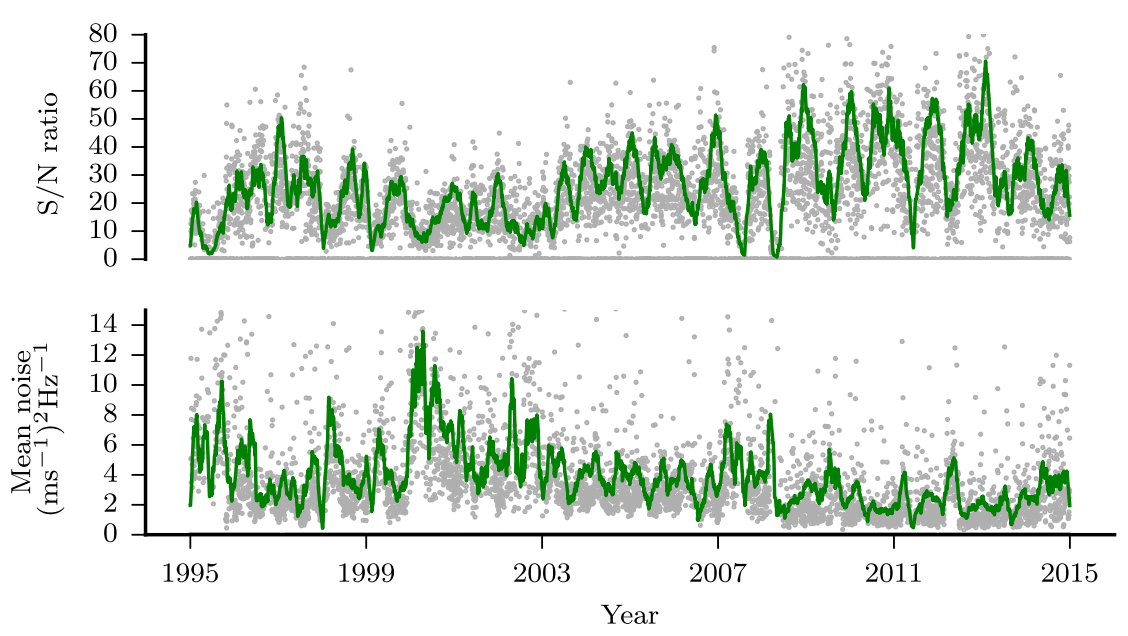

Figure 7 Sutherland data quality as a function of date. Top: Signal-to-noise ratio, higher is better. Bottom: Mean noise level, lower is better. There is one grey dot per day, and the solid green curve represents a 50-day moving mean.

weather conditions deteriorated with a heavy snow fall that left the observatory cut off by road, without internet or phone connections, and dependent on old diesel generators for electrical power (Miller, 1997). As the snow melted, the $4.2 \mathrm{kV}$ step-down transformer for the BiSON dome was first flooded and subsequently destroyed by another lightning strike. The dome was without power for more than a month, until early November 1997.

Two further visits to finalise repairs were needed in November 1997 (Lines, 1998) and January 1998 (Miller, 1998), which were related to problems with the computer, the dome azimuth motor, and the water-loop system that stabilises the instrumentation temperatures. Noise problems in both detectors persisted until early 1999, clearly visible in the plot of data quality (Figure 9). A large part of 2000 was lost due to a broken declination gearbox on the mount and also to the failure of the dome azimuth motor. More electrical problems occurred in May 2014, when faults with both the shutter and blind limit switches caused the circuit breakers to trip repeatedly. A site visit was required to replace the limit switches and also work on additional problems with the water-loop pump and the uninterruptable power supplies (Hale, 2014b).

Las Campanas is the best-performing station in the network, consistently supplying duty cycles above $80 \%$ in the summer and regularly above $40 \%$ even during the winter months. From 2012 the noise performance has deteriorated slightly. A recent site visit indicated reduced performance from the potassium vapour cell, and this may need to be replaced soon.

\subsection{Narrabri, NSW, Australia}

The final fully automated site was installed in Narrabri, Australia, in 1992 (Williams, 2004). It is on the site of the Australia Telescope Compact Array (ATCA) at the Paul Wild Observatory operated by The Commonwealth Scientific and Industrial Research Organisation (CSIRO). The observatory is around $550 \mathrm{~km}$ north-west of Sydney.

Narrabri received its Zoo upgrade in June 2004 (Jackson and Miller, 2004), new temperature controllers in January 2010 (Barnes and Hale, 2010; Barnes, 2010), and a new 

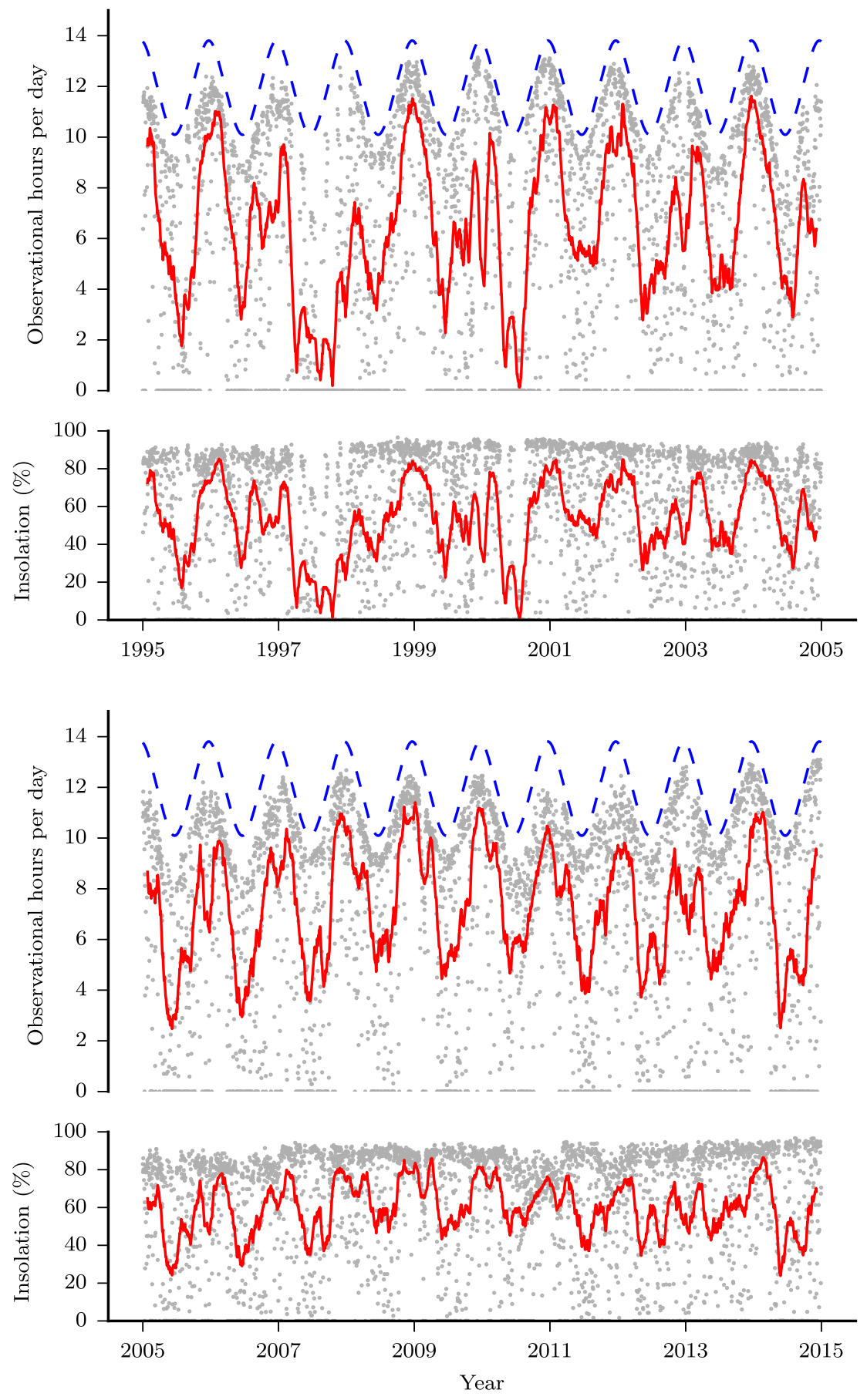

Figure 8 Las Campanas duty cycle as a function of date, plotted in hours per day, and as a percentage of potential daylight hours. There is one grey dot per day, and the solid red curve represents a 50-day moving mean. The dashed blue curve shows potential daylight hours. 


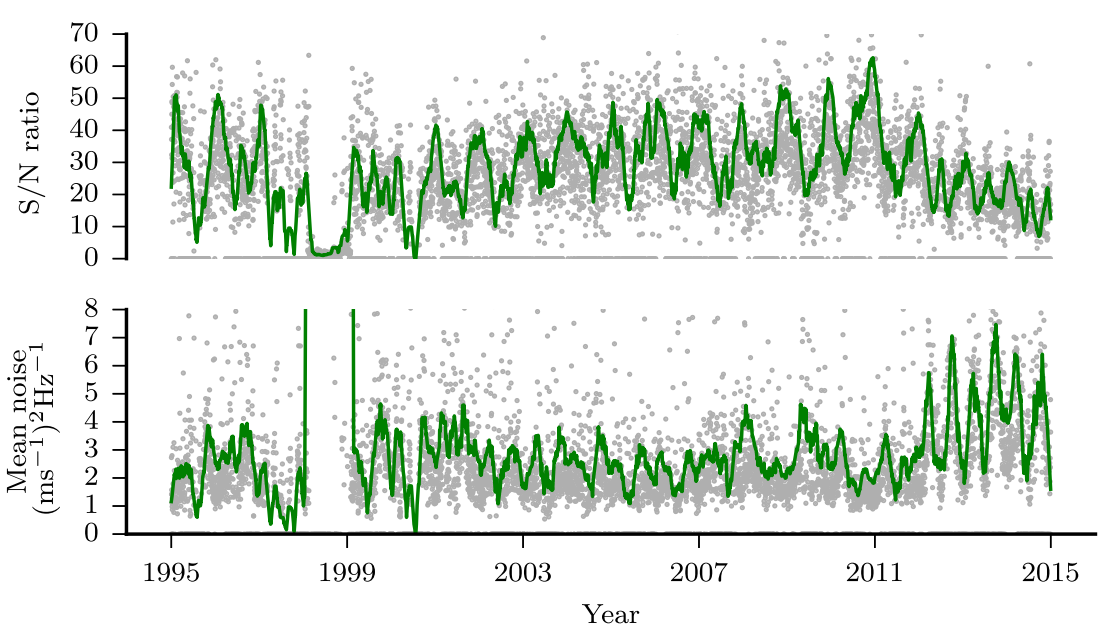

Figure 9 Las Campanas data quality as a function of date. Top: Signal-to-noise ratio, higher is better. Bottom: Mean noise level, lower is better. There is one grey dot per day, and the solid green curve represents a 50-day moving mean.

digital autoguider and counters in April 2013 (Hale and Davies, 2013). In February 2000, the dome blind motor failed (Miller, 2000) and was replaced with an identical spare from Birmingham, but failed again in March 2003, by which time the part had been discontinued. The dome manufacturer specified an alternative part for the blind mechanism, and this was installed in July 2003 (New and Isaak, 2003). Following several further motor failures, a change to the control system was identified as being required due to differences in the motor type (Jackson and Miller, 2004).

The Narrabri duty cycle is shown in Figure 10. The gap in 1997 is due to problems with the scalers and correct termination of the signal cables from the voltage-to-frequency output of the detectors. The missing data in 2003 and 2004 are due to the blind motor problems discussed earlier. In 2013 a fault on the anemometer in June caused problems with the weather module, and this kept the dome closed unnecessarily.

In the plot of data quality, Figure 11, the increased noise level in 2003 is due to a failure of an interference filter temperature controller, and in 2009 is due to a faulty power supply producing under-voltage power rails. Other than these faults, the site shows solid performance.

\subsection{Mount Wilson, California, USA}

The final change to arrive at the existing network configuration was made in 1992. The Mark III instrument was moved from Hawaii to the 60-foot tower at the Mount Wilson Hale Observatory in California (Elsworth, 1992). This is the very same observatory where Robert Leighton originally discovered the solar five-minute oscillations (Leighton, Noyes, and Simon, 1962). The observatory is located in the San Gabriel Mountains near Pasadena, around $80 \mathrm{~km}$ north-east of Los Angeles. The tower is operated by Ed Rhodes and his team of undergraduate volunteer observers. Like Izaña, the tower uses a cœlostat to direct light down the tower into the observing room below, and as such requires someone to open the dome and align the mirrors each morning. It also requires presence throughout the day to close the dome in the event of bad weather and at the end of the observing session. 

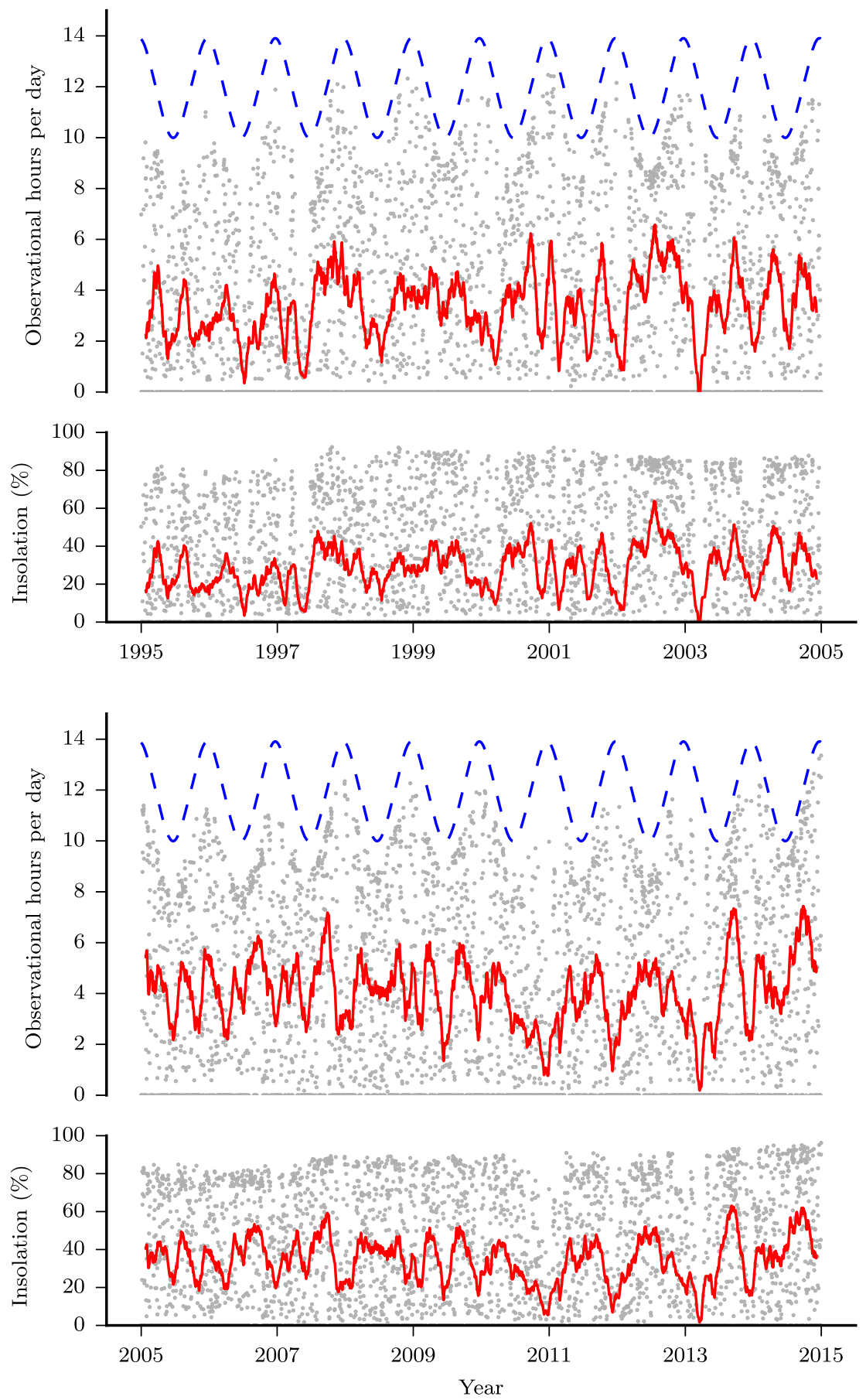

Figure 10 Narrbri duty cycle as a function of date, plotted in hours per day, and as a percentage of potential daylight hours. There is one grey dot per day, and the solid red curve represents a 50-day moving mean. The dashed blue curve shows potential daylight hours. 

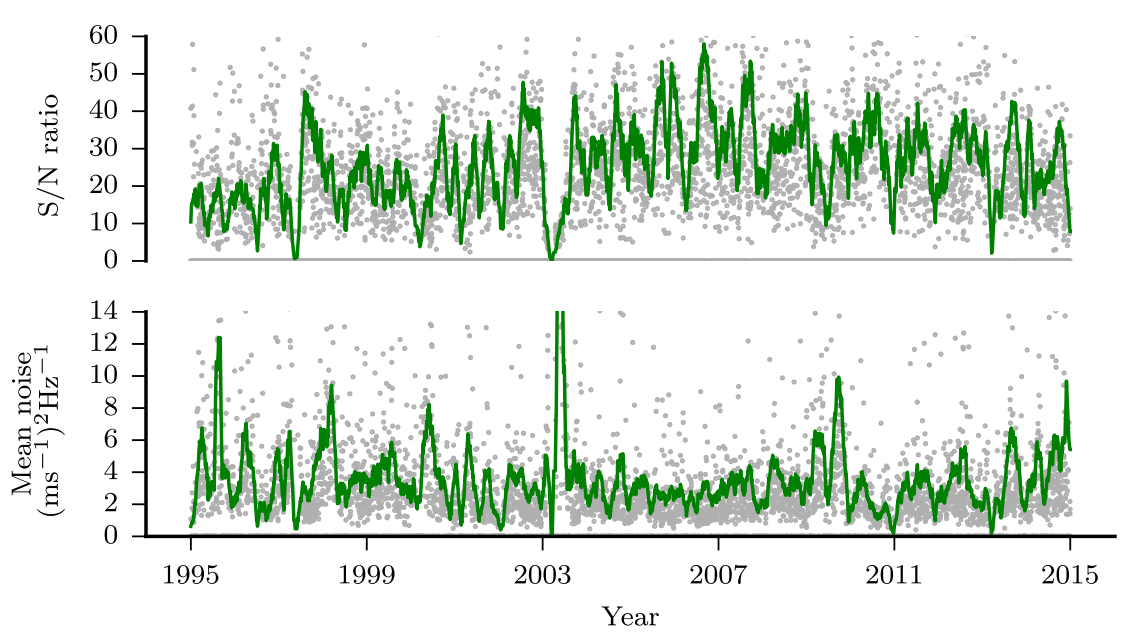

Figure 11 Narrabri data quality as a function of date. Top: Signal-to-noise ratio, higher is better. Bottom: Mean noise level, lower is better. There is one grey dot per day, and the solid green curve represents a 50-day moving mean.

The original optical configuration used by Leighton involved the colostat firing light 60 feet down the tower, a further 30 feet down into a pit where it would reflect from the spectroheliograph, and finally another 30 feet back up the pit to the observing room above, the total optical path length being 120 feet. The spectroheliograph is no longer in use, and so the current optical configuration is somewhat different. The primary instrument operated by Rhodes used a small objective lens near the base of the tower. Since it required only half the original optical path length, the colostat mirrors are now effectively oversized for their current usage. We can take advantage of this by picking off a small section of the beam and directing it to another instrument without affecting the operation of the main instrument. This is done using a periscope arrangement of mirrors mounted in the tower shaft taking a part of the beam and shifting it slightly to the south where it is directed back down the tower. Another mirror at the bottom reflects the light horizontally into the BiSON spectrometer. In total, five mirrors are used to direct light into the spectrometer.

There are regular gaps in the data at midday during the winter months, when the primary mirror has to be moved from the east side of the tower in the morning to the west in the afternoon, in order to avoid shadowing from the secondary mirror. Unlike Izaña, the secondary at Mount Wilson has only one mounting configuration. The Mount Wilson duty cycle is shown in Figure 12.

In July 1996 the Mark III instrument from Haleakala was retired and replaced with the tenth spectrometer designed by the group, code-named Klaus (Miller and New, 1999). The new design offered much-improved data quality in line with the other newer instruments in the network, and the reduction in noise level can be seen clearly in the plot of data quality (Figure 13).

The optical configuration with the pick-off mirrors is marginal, and very precise alignment is required for optimum performance of the spectrometer. Aligning five different mirrors over such large distances and ensuring no vignetting is difficult. Vibrations from the tower can also cause the mirrors or the spectrometer itself to move slightly, meaning the optical alignment has to be checked frequently. The large step in noise level during 2004 corresponds to an autoguider problem, where it failed to drive in right ascension. Mount 

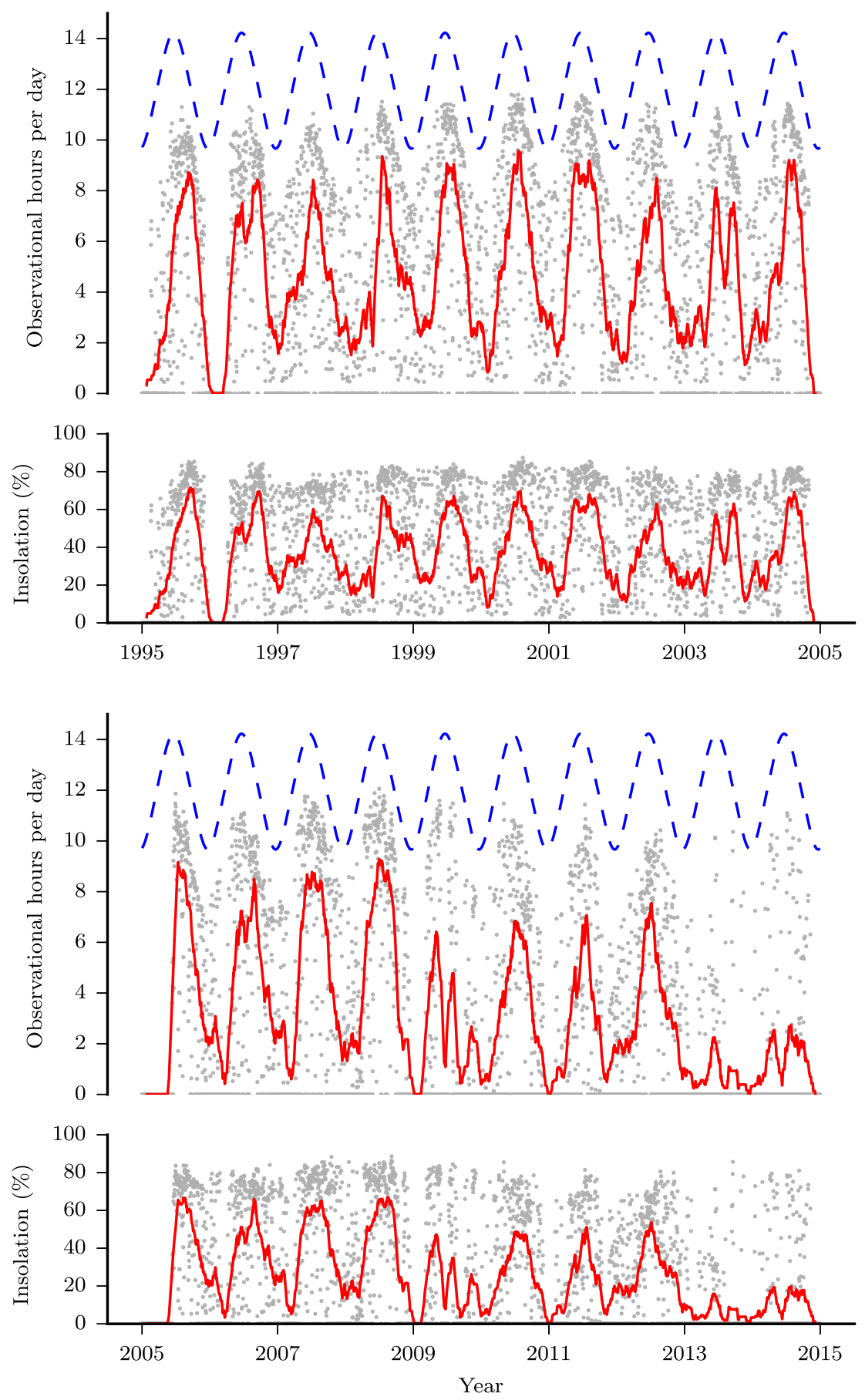

Figure 12 Mount Wilson duty cycle as a function of date, plotted in hours per day, and as a percentage of potential daylight hours. There is one grey dot per day, and the solid red curve represents a 50-day moving mean. The dashed blue curve shows potential daylight hours. 

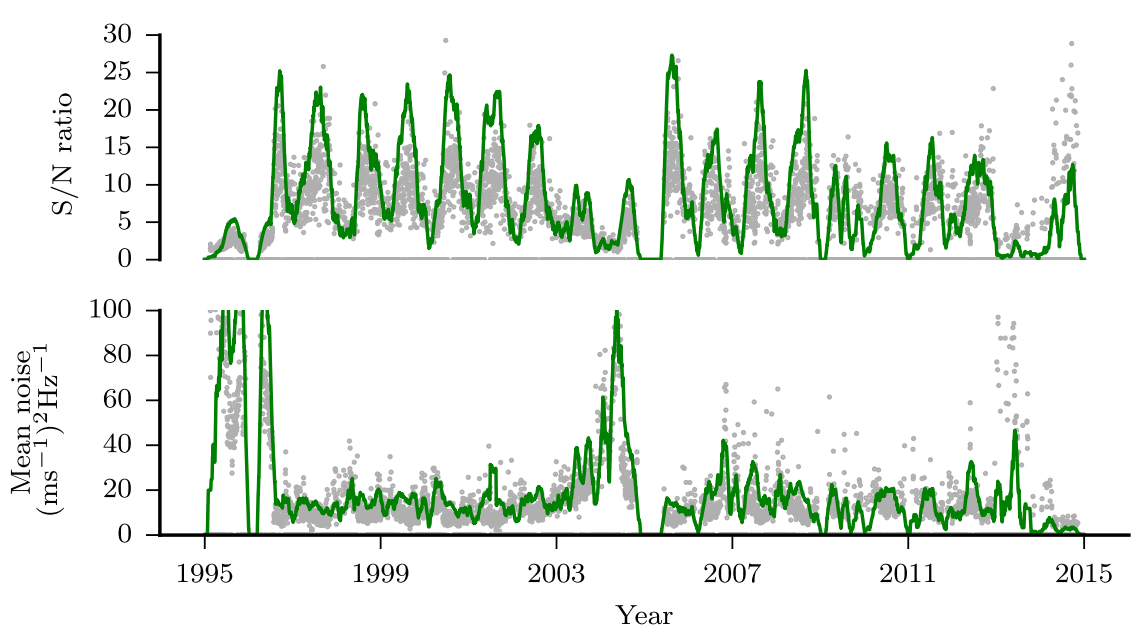

Figure 13 Mount Wilson data quality as a function of date. Top: Signal-to-noise ratio, higher is better. Bottom: Mean noise level, lower is better. There is one grey dot per day, and the solid green curve represents a 50-day moving mean. The large step in 1996 is due to installation of a new upgraded spectrometer, and in 2004 due to autoguider problems.

Wilson received its Zoo upgrade in September 2005 (Miller, 2005), which included alignment of all the optics returning the site to its original performance. In 2009 a variety of faults caused loss of data. The computer failed at the beginning of the year, and a site visit to replace it could not be arranged until March. Shortly after installing the new computer, the UPS failed in May and caused problems with the temperature controller. The on-site help investigated the problem, but could not locate the fault. Another site visit was arranged for July, where a blown fuse was found and the problem solved. At the end of July, the mirrors were removed for two weeks for re-aluminisation. Just at the point where everything was working, in August the entire observatory was evacuated due to the nearby Station Fire. There was concern that the entire observatory could be lost. Fortunately, that was not the case, thanks to efforts from the fire service and the US Forest Service.

Further guider problems were experienced in 2011, where one of the motors failed again and needed to be replaced. Through 2013 and 2014, significant problems were experienced with the autoguider electronics in the tower. On a site visit from Birmingham, the guider system built into the second flat of the colostat was completely rewired and refurbished, removing some very old and corroded cables and producing a significant decrease in noise level (Hale, 2014a). Significant damage to the primary mirror was also discovered during the visit. This is a serious problem since it is implicit in our analysis that we see the whole of the solar disc and that no part is vignetted. Since the Sun is rotating, any vignetting of the disc causes uneven weighting and produces an offset in the computed residuals through a process known as Doppler Imaging (Brookes, Isaak, and van der Raay, 1978b). For the Sun, when viewing a typical Fraunhofer line, the ratio in weighting of opposite sides of the disc is about 4:1. The instrument used at Mount Wilson is the same design as used at our other sites on equatorial mounts. It is optically designed to directly observe the Sun. At Mount Wilson, the cœlostat is approximately 60 feet away from the spectrometer, at the top of the tower. Optically this is near infinity, and so the spectrometer forms an image of the surface of the colostat mirrors, with the result that it is essential the mirrors be clean and in good condition. The damaged area of the mirror was causing part of the solar disc to be missing 
from the light entering the spectrometer. The problem was compounded due to the unusual design of the tower cœlostat. Usually, the primary mirror performs both tracking and guiding to follow the Sun throughout the day. The Mount Wilson cœlostat separates these functions between the two mirrors - the primary mirror tracks whilst the secondary mirror guides. This means that any dirt or damage on the surface of the primary mirror will give rise to a signal that will be seen to oscillate at the frequency of the tracking error, as the autoguider on the secondary mirror compensates for tracking errors of the primary mirror. The worm-drive of the primary mirror uses 432 teeth, which puts the expected gear frequency at $2.5 \mathrm{mHz}$. This causes the vignetted part of the solar image to oscillate at $2.5 \mathrm{mHz}$, periodically varying the weighting of the solar disc, and corrupting the power spectrum at this frequency with sufficient amplitude to make the data unusable. Unfortunately, it is almost impossible to filter out this fault since it is within the main solar five-minute-oscillation band of interest, falling very close to one of the mode peaks and is not sufficiently coherent to be removed by subtracting a single sine wave. The fault was able to be mitigated by rotating the mirror in its housing to move most of the damage to an area not used by our pick-off mirrors. A similar fault occurs if the two mirrors are not aligned correctly at the start of an observing period. If the light from the primary mirror falls off the edge of the secondary mirror, then again the solar disc is vignetted and the $2.5 \mathrm{mHz}$ tracking error becomes visible.

\section{Whole Network Performance}

Plots of the whole-network duty cycle and data window-function are shown in Figures 14 and 15 . In a total of 7305 days, $45.5 \%$ of the available time was covered by one site, $30.3 \%$ by two sites, $6 \%$ by three sites, $0.06 \%$ by four simultaneous sites. The horizontal lines that run from 1995-2005 in Figure 15 are caused by a midday beam-chopper used to check the dark counts from the detectors during the day. From 2006 it was decided that this was no longer necessary, and so the regular gap is not present. Other regular gaps are those caused by cœlostat shadowing at Izaña and Mount Wilson, as discussed in Section 5. Figure 16 shows a histogram of the daily fill. Just 9 days had no coverage, and 633 days have a fill greater than $99 \%$ of which 312 days achieved $100 \%$. The average fill is $82 \%$ for the whole dataset.

The original data pipeline for calibration of raw data from the BiSON spectrometers through to velocity residuals is described by Elsworth et al. (1995). The next stage of analysis involves combining the residuals into an extended time series and transforming into the frequency domain where the mode characteristics can be analysed. This is described by Chaplin et al. (1997) and Hale (2003). An updated pipeline that includes correction for differential atmospheric extinction was produced by Davies et al. (2014a). By applying differential extinction correction, we have removed most of the low-frequency drifts in the dataset that were previously filtered using a 25 -sample moving mean, and this allows further investigation of the very low frequency modes of oscillation that were previously lost in the noise background. We are now also able to make better use of weighted averaging of overlapping sites to produce a further improvement in the signal-to-noise ratio.

The entire network archive of velocity residuals has been regenerated using the latest data pipeline, and a new concatenated time series has been produced for the period from 1995 to the end of 2014 using the latest weighted-averaging techniques. A noise ceiling of $100\left(\mathrm{~m} \mathrm{~s}^{-1}\right)^{2} \mathrm{~Hz}^{-1}$ was selected to reject data above this level, and this has reduced the overall fill from $82 \%$ to $78 \%$. The plot of data quality and noise levels (Figure 17) shows 

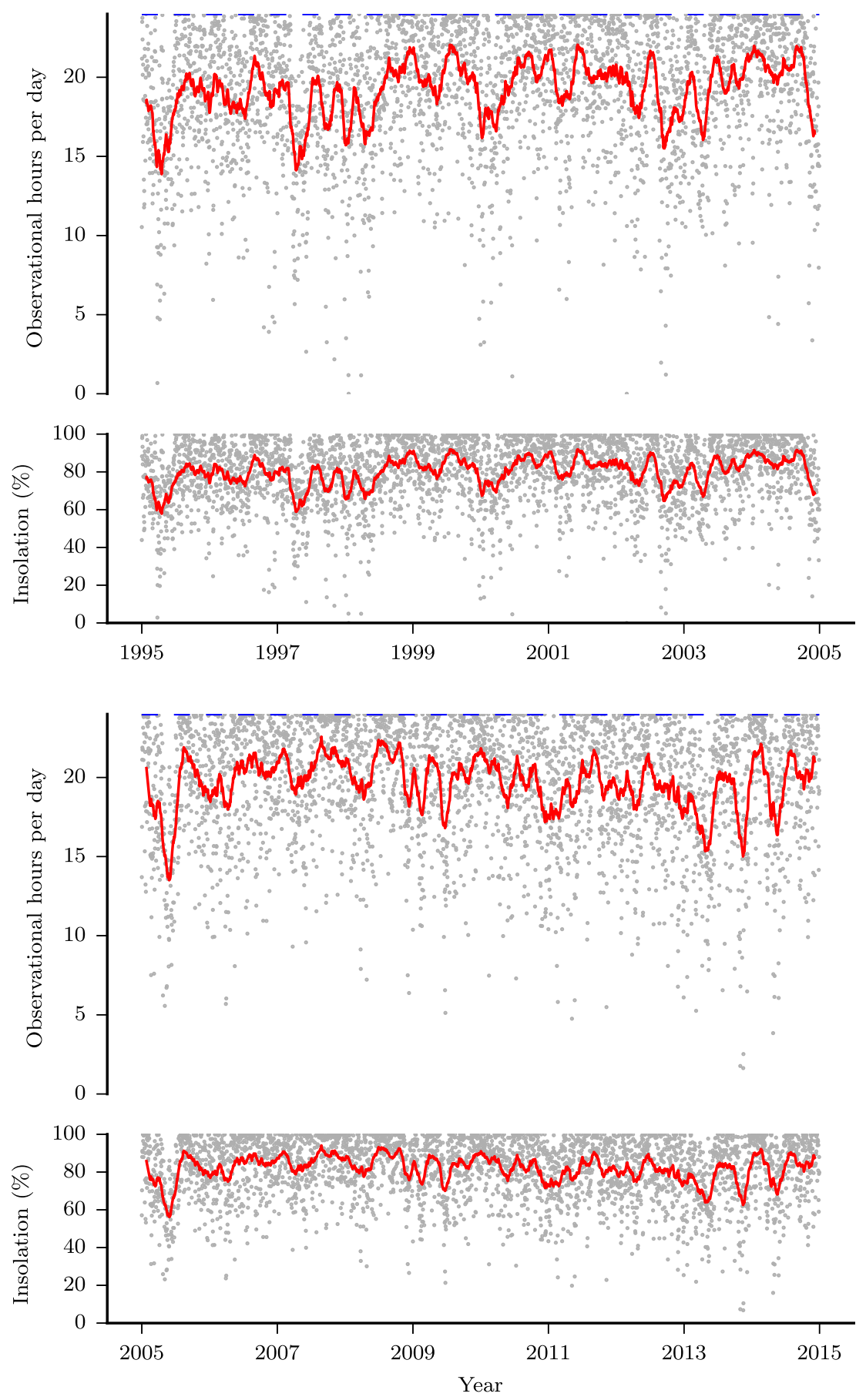

Figure 14 All-station duty cycle as a function of date, plotted in hours per day, and as a percentage of potential daylight hours. There is one grey dot per day, and the red curve represents a 50-day moving mean. 

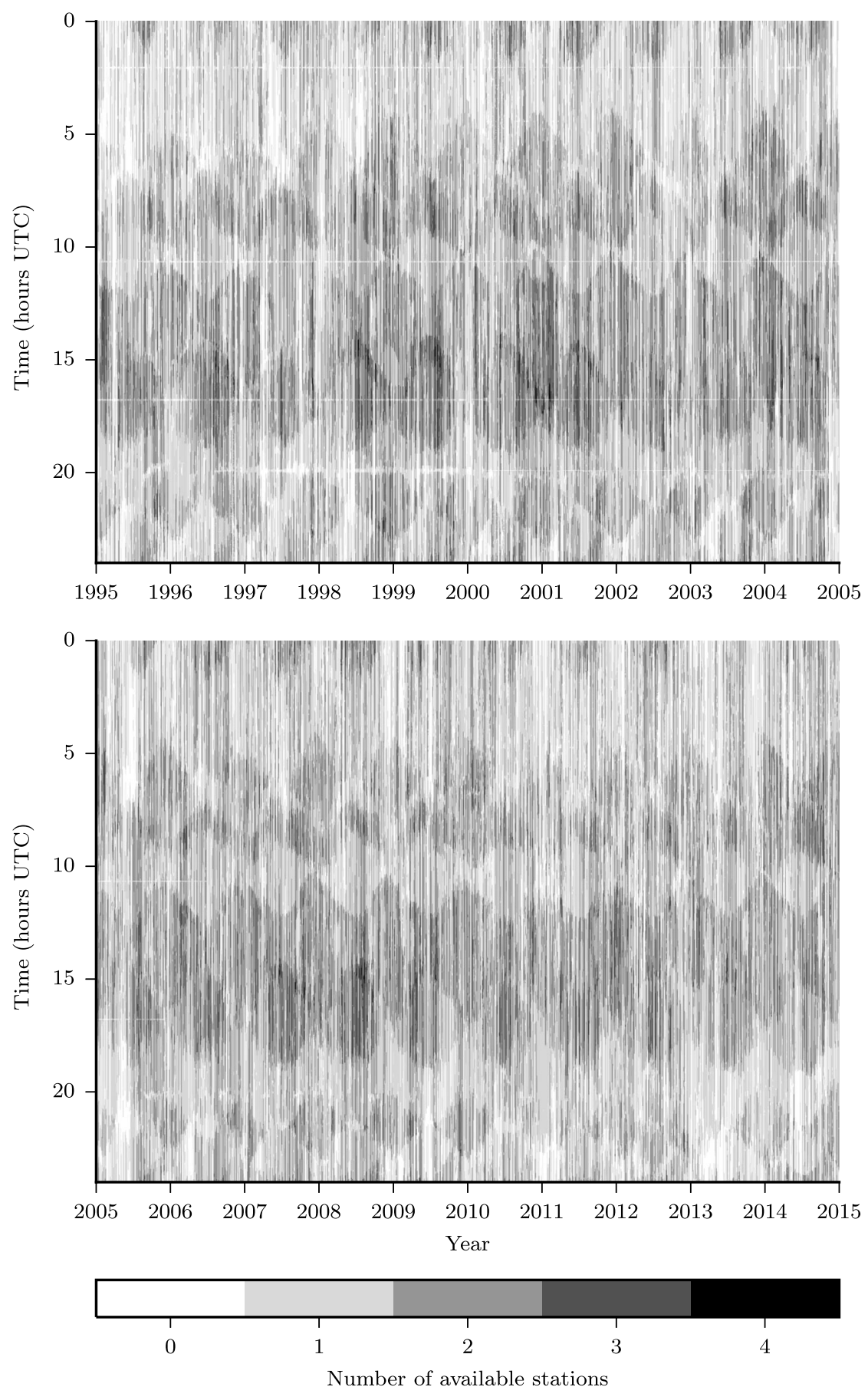

Figure 15 All-station data window-function. 


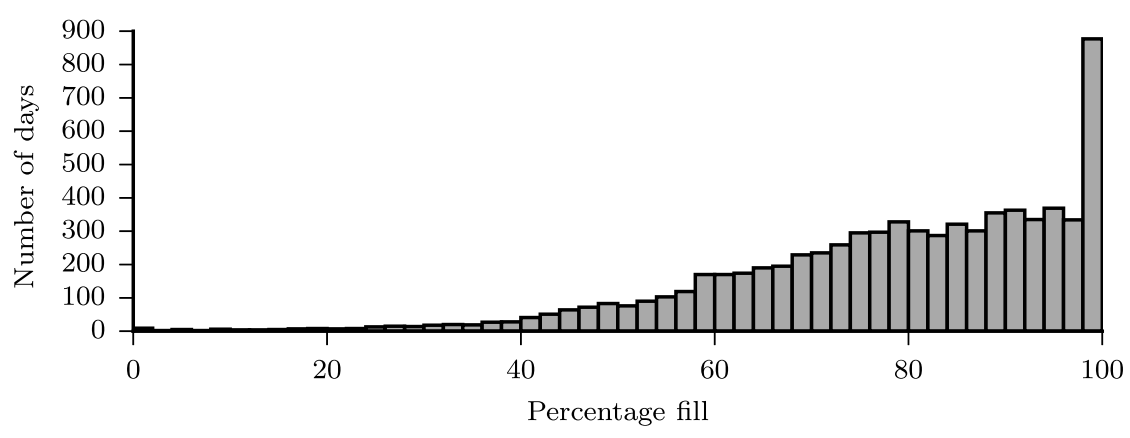

Figure 16 The distribution of fill per day for the overall time series.
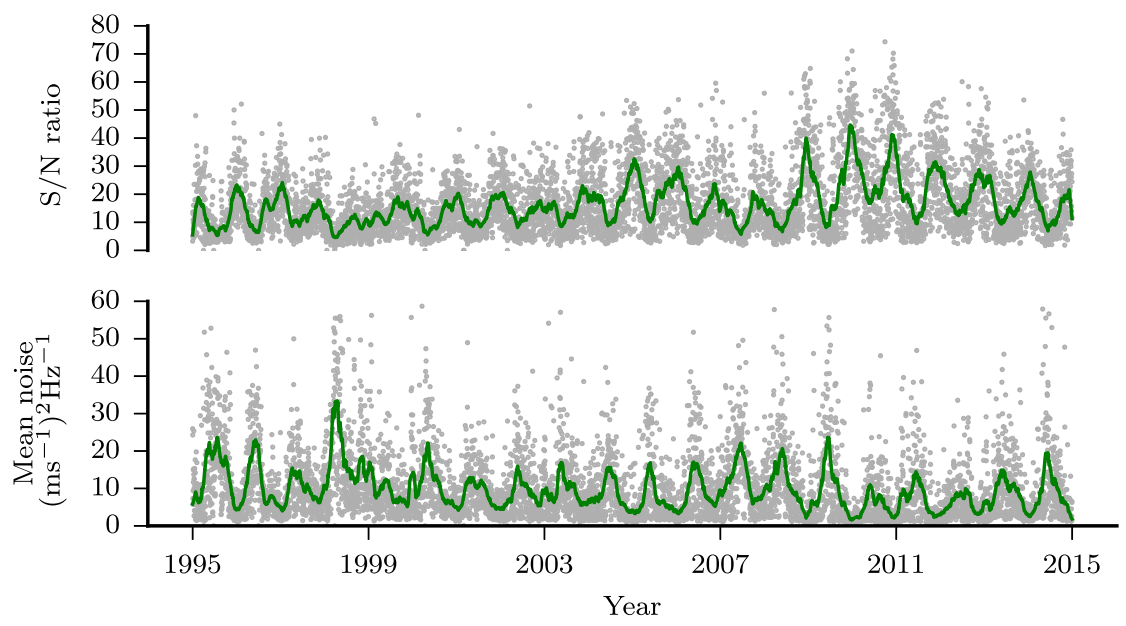

Figure 17 All station data quality as a function of date. Top: Signal-to-noise ratio, higher is better. Bottom: Mean noise level, lower is better. There is one grey dot per day, and the solid green curve represents a 50-day moving mean.

excellent stability through the entire period. Histograms of noise level and FOM (Figures 18 and 19) show the daily distribution of data quality.

The new data pipeline provides an improvement across the entire historic archive, not just new data, and as such provides an exciting opportunity for new science.

\section{BiSON Open Data Portal}

All data produced by the BiSON are freely available from the BiSON Open Data Portal http://bison.ph.bham.ac.uk/opendata - and are also in the process of being deposited in the University of Birmingham Long Term Storage Archive (LTSA). The LTSA ensures the data will be available via a persistent URL for a minimum of ten years. Data will be available from the archive using the "FindIt@Bham" service - http://findit.bham.ac.uk - sand we also hope to provide all datasets with a Digital Object Identifier (DOI) as soon as this facility becomes available via the archive. 


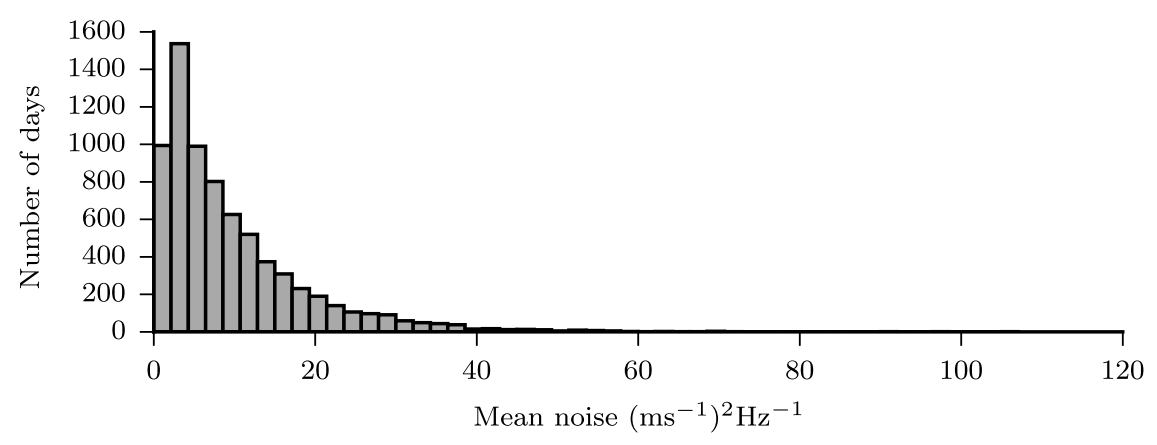

Figure 18 The distribution of mean noise per day for the overall time series. Lower is better.

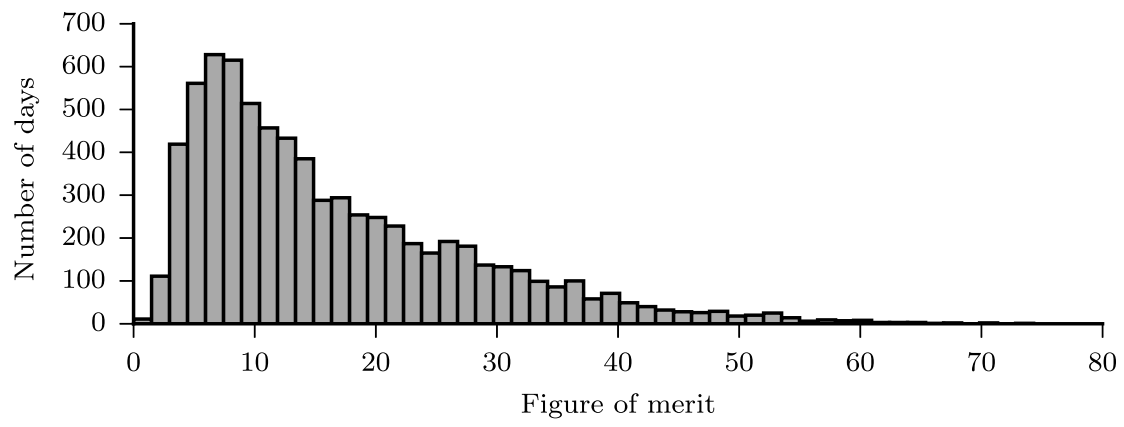

Figure 19 The distribution of FOM per day for the overall time series. Higher is better.

Data products are in the form of calibrated velocity residuals, concatenated into a single time series from all BiSON sites. Individual days of data, and also bespoke products produced from requested time periods and sites, are available by contacting the authors. We hope to also provide all raw data products via the LTSA as the archive is populated. Oscillation mode frequencies and amplitudes are available from Broomhall et al. (2009) and Davies et al. (2014b).

All data created specifically during the research for this article are openly available from the University of Birmingham ePapers data archive (Hale, 2015a) and are also listed on the BiSON Open Data Portal.

\section{To the Future}

It would be scientifically advantageous to increase the network duty cycle from $78 \%$ to $100 \%$ or better (i.e. to have every $40 \mathrm{~s}$ interval covered by one or more sites), and also to make better use of weighted averaging of multi-site data. We saw in Section 5 a seasonal variation in noise level caused by the changing Doppler offset throughout the year. A similar offset effect is seen on a daily period due to Earth's rotation, and this causes instruments at different longitudes to sample line formation at different heights in the solar atmosphere, meaning that the noise between sites is not completely coherent. Simultaneous observing at as many sites as possible allows the incoherent components of the noise to be beaten 
down and potentially gives access to solar $g$-modes, which are expected to have very low frequencies and low amplitudes (Appourchaux et al., 2010).

Technology has moved on significantly since the BiSON nodes were designed in the late 1980s. Whilst a considerable programme of upgrades has taken place over the years, the overall design is still limited by the original specification. Deploying more nodes in the classic style would be prohibitively expensive.

By taking advantage of modern fiber optics and electronic miniaturisation such as microcontrollers and single-board computers, it is possible to design a solar spectrometer with a much smaller physical footprint and considerably lower deployment cost, thus making it feasible to observe from many more sites. Work is underway on a second-generation network that will operate as a complement to the existing nodes, and this aims to guarantee better than $100 \%$ duty cycle and much lower noise levels.

Disclosure of Potential Conflicts of Interest The authors declare that they have no conflicts of interest.

Acknowledgements We would like to thank all those who are, or have been, associated with BiSON. In Birmingham: George Isaak, Bill Brookes, Bob van der Raay, Clive McLeod, Roger New, Sarah Wheeler, Clive Speake, Brek Miller, Richard Lines, Phil Pavelin, Barry Jackson, Hugh Williams, Joe Litherland, Ian Barnes, Richard Bryan, and John Allison. In Mount Wilson: Ed Rhodes, Stephen Pinkerton, the team of USC undergraduate observing assistants, former USC staff members Maynard Clark, Perry Rose, Natasha Johnson, Steve Padilla, and Shawn Irish, and former UCLA staff members Larry Webster and John Boyden. In Las Campanas: Patricio Pinto, Andres Fuentevilla, Emilio Cerda, Frank Perez, Marc Hellebaut, Patricio Jones, Gastón Gutierrez, Juan Navarro, Francesco Di Mille, Roberto Bermudez, and the staff of LCO. In Izaña: We would like to give particular thanks to Pere Pallé and Teo Roca Cortés, and all staff at the IAC who have contributed to running the Mark I instrument over many years (see also the acknowledgements in Roca Cortés and Pallé, 2014). In Sutherland: Pieter Fourie, Willie Koorts, Jaci Cloete, Reginald Klein, John Stoffels, and the staff of SAAO. In Carnarvon: Les Bateman, Les Schultz, Sabrina Dowling-Giudici, Inge Lauw of Williams and Hughes Lawyers, and NBN Co. Ltd. In Narrabri: Mike Hill and the staff of CSIRO. BiSON is funded by the Science and Technology Facilities Council (STFC).

Open Access This article is distributed under the terms of the Creative Commons Attribution 4.0 International License (http://creativecommons.org/licenses/by/4.0/), which permits unrestricted use, distribution, and reproduction in any medium, provided you give appropriate credit to the original author(s) and the source, provide a link to the Creative Commons license, and indicate if changes were made.

\section{References}

Appourchaux, T., Belkacem, K., Broomhall, A.-M., Chaplin, W.J., Gough, D.O., Houdek, G., Provost, J., Baudin, F., Boumier, P., Elsworth, Y., García, R.A., Andersen, B.N., Finsterle, W., Fröhlich, C., Gabriel, A., Grec, G., Jiménez, A., Kosovichev, A., Sekii, T., Toutain, T., Turck-Chièze, S.: 2010, The quest for the solar g modes. Astron. Astrophys. Rev. 18, 197. DOI. ADS.

Barnes, I.: 2008, The installation of the Sutherland temperature controllers in 2007 August. BiSON Technical Report Series 294, High-Resolution Optical-Spectroscopy Group, University of Birmingham, UK. http://epapers.bham.ac.uk/2036/.

Barnes, I.: 2009, The Sutherland temperature controllers. BiSON Technical Report Series 316, HighResolution Optical-Spectroscopy Group, University of Birmingham, UK. http://epapers.bham.ac.uk/ 2040/.

Barnes, I.: 2010, The Narrabri temperature controller. BiSON Technical Report Series 333, High-Resolution Optical-Spectroscopy Group, University of Birmingham, UK. http://epapers.bham.ac.uk/2047/.

Barnes, I., Hale, S.J.: 2005, Carnarvon trip report - May 2005. BiSON Technical Report Series 253, HighResolution Optical-Spectroscopy Group, University of Birmingham, UK. http://epapers.bham.ac.uk/ 2026/.

Barnes, I., Hale, S.J.: 2010, The installation of new temperature controllers at Narrabri in 2010 February. BiSON Technical Report Series 332, High-Resolution Optical-Spectroscopy Group, University of Birmingham, UK. http://epapers.bham.ac.uk/2046/. 
Barnes, I., Jackson, B., Miller, B.A.: 2003, BeebSub \#56A and BeebSub \#56B - BBC computer substitutes. BiSON Technical Report Series 218, High-Resolution Optical-Spectroscopy Group, University of Birmingham, UK. http://epapers.bham.ac.uk/2014/.

Barnes, I., Jackson, B., Miller, B.A.: 2004, BeebSub \#56C - An improved BBC computer substitute. BiSON Technical Report Series 243, High-Resolution Optical-Spectroscopy Group, University of Birmingham, UK. http://epapers.bham.ac.uk/2023/.

Barnes, I., Miller, B.A.: 2006, The grand opening of the Sutherland Zoo. BiSON Technical Report Series 276, High-Resolution Optical-Spectroscopy Group, University of Birmingham, UK. http://epapers. bham.ac.uk/2032/.

Barnes, I., Miller, B.A.: 2009, Jabba is returned to Carnarvon in 2009 July. BiSON Technical Report Series 323, High-Resolution Optical-Spectroscopy Group, University of Birmingham, UK. http://epapers. bham.ac.uk/2043/.

Barnes, I., Miller, B.A.: 2011, The mount controller: A digital autoguider for Las Campanas. BiSON Technical Report Series 344, High-Resolution Optical-Spectroscopy Group, University of Birmingham, UK. http://epapers.bham.ac.uk/2049/.

Barnes, I., Miller, B.A., Jackson, B.: 2007, The removal of Jabba from Carnarvon in 2006 November. BiSON Technical Report Series 282, High-Resolution Optical-Spectroscopy Group, University of Birmingham, UK. http://epapers.bham.ac.uk/2034/.

Brookes, J.R., Isaak, G.R., van der Raay, H.B.: 1976, Observation of free oscillations of the sun. Nature 259, 92. DOI. ADS.

Brookes, J.R., Isaak, G.R., van der Raay, H.B.: 1978a, A resonant-scattering solar spectrometer. Mon. Not. Roy. Astron. Soc. 185, 1. ADS.

Brookes, J.R., Isaak, G.R., van der Raay, H.B.: 1978b, The observation of a rotating body using highresolution spectroscopy. Mon. Not. Roy. Astron. Soc. 185, 19. ADS.

Broomhall, A.-M., Chaplin, W.J., Davies, G.R., Elsworth, Y., Fletcher, S.T., Hale, S.J., Miller, B., New, R.: 2009, Definitive Sun-as-a-star p-mode frequencies: 23 years of BiSON observations. Mon. Not. Roy. Astron. Soc. 396, L100. DOI. ADS.

Chaplin, W.J., Elsworth, Y., Howe, R., Isaak, G.R., McLeod, C.P., Miller, B.A., van der Raay, H.B., Wheeler, S.J., New, R.: 1996, BiSON performance. Solar Phys. 168, 1. DOI. ADS.

Chaplin, W.J., Elsworth, Y., Howe, R., Isaak, G.R., McLeod, C.P., Miller, B.A., New, R.: 1997, Techniques used in the analysis of data collected by the Birmingham Solar-Oscillations Network (BiSON). II. Frequency domain analysis \& data merging. Astron. Astrophys. Suppl. 125, 195. DOI. ADS.

Chaplin, W.J., Elsworth, Y., Isaak, G.R., Miller, B.A., New, R., Pintér, B.: 2004, Novel techniques for the identification of noise contributions to full-disc helioseismic power spectra. In: Danesy, D. (ed.) SOHO 14 Helio- and Asteroseismology: Towards a Golden Future, ESA SP 559, 360. ADS.

Chaplin, W.J., Elsworth, Y., Isaak, G.R., Miller, B.A., New, R., Pintér, B.: 2005, Noise characteristics of full-disc helioseismic observations made by resonant scattering spectrometers. Mon. Not. Roy. Astron. Soc. 359, 607. DOI. ADS.

Chou, D.-Y., Sun, M.-T., Huang, T.-Y., Lai, S.-P., Chi, P.-J., Ou, K.-T., Wang, C.-C., Lu, J.-Y., Chu, A.-L., Niu, C.-S., Mu, T.-M., Chen, K.-R., Chou, Y.-P., Jimenez, A., Rabello-Soares, M.C., Chao, H., Ai, G., Wang, G.-P., Zirin, H., Marquette, W., Nenow, J.: 1995, Taiwan Oscillation Network. Solar Phys. 160, 237. DOI. ADS.

Claverie, A., Isaak, G.R., McLeod, C.P., van der Raay, H.B., Cortes, T.R.: 1979, Solar structure from global studies of the 5-minute oscillation. Nature 282, 591. DOI. ADS.

Davidson, C., Williams, H.K.: 2004, Progress report on the construction of the South African dome 1989 November/December. BiSON Technical Report Series 226, High-Resolution Optical-Spectroscopy Group, University of Birmingham, UK. http://epapers.bham.ac.uk/2017/.

Davies, G.R., Chaplin, W.J., Elsworth, Y., Hale, S.J.: 2014a, BiSON data preparation: A correction for differential extinction and the weighted averaging of contemporaneous data. Mon. Not. Roy. Astron. Soc. 441, 3009. DOI. ADS.

Davies, G.R., Broomhall, A.M., Chaplin, W.J., Elsworth, Y., Hale, S.J.: 2014b, Low-frequency, low-degree solar p-mode properties from 22 years of Birmingham Solar Oscillations Network data. Mon. Not. Roy. Astron. Soc. 439, 2025. DOI. ADS.

Domingo, V., Fleck, B., Poland, A.I.: 1995, The SOHO mission: An overview. Solar Phys. 162, 1. DOI. ADS.

Elsworth, Y.: 1992, Update on my report on the trip to Mount Wilson (Hale) Observatory. BiSON Technical Report Series 5, High-Resolution Optical-Spectroscopy Group, University of Birmingham, UK. http://epapers.bham.ac.uk/2003/.

Elsworth, Y., Howe, R., Isaak, G.R., McLeod, C.P., Miller, B.A., New, R., Wheeler, S.J.: 1995, Techniques used in the analysis of solar oscillations data from the BiSON (University of Birmingham) network. I. Daily calibration. Astron. Astrophys. Suppl. 113, 379. ADS. 
Fossat, E.: 1991, The IRIS network for full disk helioseismology - Present status of the programme. Solar Phys. 133, 1. DOI. ADS.

Fossat, E., IRIS Group: 2002, 11 years of IRIS network exploitation. In: Combes, F., Barret, D. (eds.) $S F 2 A-$ 2002: Semaine de l'Astrophysique Francaise, EDP Sciences, Les Ulis, 521. ADS.

Fröhlich, C., Romero, J., Roth, H., Wehrli, C., Andersen, B.N., Appourchaux, T., Domingo, V., Telljohann, U., Berthomieu, G., Delache, P., Provost, J., Toutain, T., Crommelynck, D.A., Chevalier, A., Fichot, A., Däppen, W., Gough, D., Hoeksema, T., Jiménez, A., Gómez, M.F., Herreros, J.M., Cortés, T.R., Jones, A.R., Pap, J.M., Willson, R.C.: 1995, VIRGO: Experiment for helioseismology and solar irradiance monitoring. Solar Phys. 162, 101. DOI. ADS.

Gabriel, A.H., Grec, G., Charra, J., Robillot, J.-M., Roca Cortés, T., Turck-Chièze, S., Bocchia, R., Boumier, P., Cantin, M., Cespédes, E., Cougrand, B., Crétolle, J., Damé, L., Decaudin, M., Delache, P., Denis, N., Duc, R., Dzitko, H., Fossat, E., Fourmond, J.-J., García, R.A., Gough, D., Grivel, C., Herreros, J.M., Lagardère, H., Moalic, J.-P., Pallé, P.L., Pétrou, N., Sanchez, M., Ulrich, R., van der Raay, H.B.: 1995, Global Oscillations at Low Frequency from the SOHO mission (GOLF). Solar Phys. 162, 61. DOI. ADS.

Grec, G., Fossat, E., Pomerantz, M.: 1980, Solar oscillations - Full disk observations from the geographic South Pole. Nature 288, 541. DOI. ADS.

Hale, S.J.: 2003, Scientific advancements in analysis of solar oscillation data. Master's thesis, School of Physics and Astronomy, University of Birmingham, UK. http://etheses.bham.ac.uk/5952/.

Hale, S.J.: 2012, Sutherland 2012 June installation of tiger counters. BiSON Technical Report Series 358, High-Resolution Optical-Spectroscopy Group, University of Birmingham, UK. http://epapers.bham. ac.uk/2053/.

Hale, S.J.: 2013, The installation of a digital autoguider in Sutherland in 2013 November. BiSON Technical Report Series 362, High-Resolution Optical-Spectroscopy Group, University of Birmingham, UK. http://epapers.bham.ac.uk/2057/.

Hale, S.J.: 2014a, Autoguider repairs at Mount Wilson in 2014 April. BiSON Technical Report Series 365, High-Resolution Optical-Spectroscopy Group, University of Birmingham, UK. http://epapers.bham. ac.uk/2060/.

Hale, S.J.: 2014b, Blind and mount controller repairs in Las Campanas in 2014 June. BiSON Technical Report Series 366, High-Resolution Optical-Spectroscopy Group, University of Birmingham, UK. http://epapers.bham.ac.uk/2061/.

Hale, S.J.: 2015a, BiSON - All sites - 1995 to 2014 - Performance check. Birmingham Solar Oscillations Network, University of Birmingham, UK. http://epapers.bham.ac.uk/1977/.

Hale, S.J.: 2015b, The Hannibal temperature controllers. BiSON Technical Report Series 372, HighResolution Optical-Spectroscopy Group, University of Birmingham, UK. http://epapers.bham.ac.uk/ 2067/.

Hale, S.J.: 2015c, New temperature controller for Hannibal in Las Campanas in 2015 April. BiSON Technical Report Series 373, High-Resolution Optical-Spectroscopy Group, University of Birmingham, UK. http://epapers.bham.ac.uk/2068/.

Hale, S.J., Davies, G.R.: 2013, The installation of a digital autoguider and tiger counters in Narrabri in 2013 April. BiSON Technical Report Series 360, High-Resolution Optical-Spectroscopy Group, University of Birmingham, UK. http://epapers.bham.ac.uk/2055/.

Hale, S.J., Miller, B.A.: 2006, The grand opening of the Las Campanas Zoo. BiSON Technical Report Series 261, High-Resolution Optical-Spectroscopy Group, University of Birmingham, UK. http://epapers. bham.ac.uk/2029/.

Harvey, J.W., Hill, F., Hubbard, R.P., Kennedy, J.R., Leibacher, J.W., Pintar, J.A., Gilman, P.A., Noyes, R.W., Title, A.M., Toomre, J., Ulrich, R.K., Bhatnagar, A., Kennewell, J.A., Marquette, W., Patron, J., Saa, O., Yasukawa, E.: 1996, The Global Oscillation Network Group (GONG) project. Science 272, 1284. DOI. ADS.

Hoyng, P.: 1989, On the sensitivity of resonant scattering spectrometers for whole-disk solar velocity oscillation measurements. Astrophys. J. 345, 1088. DOI. ADS.

Isaak, G.R.: 1961, An atomic beam spectrophotometer. Nature 189, 373. DOI. ADS.

Jackson, B., Miller, B.A.: 2004, The grand opening of the Narrabri Zoo in 2004 July. BiSON Technical Report Series 241, High-Resolution Optical-Spectroscopy Group, University of Birmingham, UK. http://epapers.bham.ac.uk/2022/.

Leibacher, J.W., Stein, R.F.: 1971, A new description of the solar five-minute oscillation. Astrophys. Lett. 7, 191. ADS.

Leighton, R.B., Noyes, R.W., Simon, G.W.: 1962, Velocity fields in the solar atmosphere. I. Preliminary report. Astrophys. J. 135, 474. DOI. ADS. 
Lines, R.: 1998, A visit to Las Campanas after the lightning strike that destroyed our step-down transformer. BiSON Technical Report Series 71, High-Resolution Optical-Spectroscopy Group, University of Birmingham, UK. http://epapers.bham.ac.uk/2005/.

McLeod, C.P.: 2002, Mark I scaler system. BiSON Technical Report Series 184, High-Resolution OpticalSpectroscopy Group, University of Birmingham, UK. http://epapers.bham.ac.uk/2009/.

Miller, B.A.: 1997, The trip to Las Campanas during the big snowstorm of 1997 August. BiSON Technical Report Series 62, High-Resolution Optical-Spectroscopy Group, University of Birmingham, UK. http://epapers.bham.ac.uk/2004/.

Miller, B.A.: 1998, A visit to Las Campanas in 1998 January. BiSON Technical Report Series 74, HighResolution Optical-Spectroscopy Group, University of Birmingham, UK. http://epapers.bham.ac.uk/ 2006/.

Miller, B.A.: 2000, The replacement of the blind motor and the connection of the station to the Internet in Narrabri in 2000 March. BiSON Technical Report Series 138, High-Resolution Optical-Spectroscopy Group, University of Birmingham, UK. http://epapers.bham.ac.uk/2008/.

Miller, B.A.: 2002, How do you get to the zoo? BiSON Technical Report Series 187, High-Resolution OpticalSpectroscopy Group, University of Birmingham, UK. http://epapers.bham.ac.uk/2010/.

Miller, B.A.: 2003, The grand opening of the Carnarvon Zoo in 2002 November. BiSON Technical Report Series 193, High-Resolution Optical-Spectroscopy Group, University of Birmingham, UK.

Miller, B.A.: 2005, The grand opening of the Mount Wilson Zoo. BiSON Technical Report Series 255, HighResolution Optical-Spectroscopy Group, University of Birmingham, UK. http://epapers.bham.ac.uk/ 2027/.

Miller, B.A.: 2011, The installation of a digital autoguider in Las Campanas in 2011 March. BiSON Technical Report Series 343, High-Resolution Optical-Spectroscopy Group, University of Birmingham, UK. http://epapers.bham.ac.uk/2048/.

Miller, B.A., New, R.: 1999, The installation of Klaus at Mount Wilson in 1996 June. BiSON Technical Report Series 106, High-Resolution Optical-Spectroscopy Group, University of Birmingham, UK. http://epapers.bham.ac.uk/2007/.

New, R., Hale, S.J.: 2006, Carnarvon trip report - July/August 2005. BiSON Technical Report Series 260, High-Resolution Optical-Spectroscopy Group, University of Birmingham, UK. http://epapers.bham. ac.uk/2028/.

New, R., Isaak, G.R.: 2003, Work carried out at Narrabri in 2003 July. BiSON Technical Report Series 213, High-Resolution Optical-Spectroscopy Group, University of Birmingham, UK. http://epapers.bham. ac.uk/2013/.

Roca Cortés, T., Pallé, P.L.: 2014, The Mark-I helioseismic experiment-I. Measurements of the solar gravitational redshift (1976-2013). Mon. Not. Roy. Astron. Soc. 443, 1837. DOI. ADS.

Salabert, D., Fossat, E., Gelly, B., Tomczyk, S., Pallé, P., Jiménez-Reyes, S.J., Cacciani, A., Corbard, T., Ehgamberdiev, S., Grec, G., Hoeksema, J.T., Kholikov, S., Lazrek, M., Schmider, F.X.: 2002a, IRIS ${ }^{++}$ database: Merging of IRIS + Mark-1 + LOWL. Astron. Astrophys. 390, 717. DOI. ADS.

Salabert, D., Jiménez-Reyes, S.J., Fossat, E., Gelly, B., Schmider, F.X.: 2002b, Variability of p-mode parameters in 11 years of IRIS ${ }^{++}$data. In: Wilson, A. (ed.) From Solar Min to Max: Half a Solar Cycle with SOHO, ESA SP 508, 95. ADS.

Scherrer, P.H., Bogart, R.S., Bush, R.I., Hoeksema, J.T., Kosovichev, A.G., Schou, J., Rosenberg, W., Springer, L., Tarbell, T.D., Title, A., Wolfson, C.J., Zayer, I., MDI Engineering Team: 1995, The solar oscillations investigation - Michelson Doppler Imager. Solar Phys. 162, 129. DOI. ADS.

Schou, J., Scherrer, P.H., Bush, R.I., Wachter, R., Couvidat, S., Rabello-Soares, M.C., Bogart, R.S., Hoeksema, J.T., Liu, Y., Duvall, T.L., Akin, D.J., Allard, B.A., Miles, J.W., Rairden, R., Shine, R.A., Tarbell, T.D., Title, A.M., Wolfson, C.J., Elmore, D.F., Norton, A.A., Tomczyk, S.: 2012, Design and ground calibration of the Helioseismic and Magnetic Imager (HMI) instrument on the Solar Dynamics Observatory (SDO). Solar Phys. 275, 229. DOI. ADS.

Stallman, R.M.: 1983, New Unix implementation. https://www.gnu.org/gnu/initial-announcement.html.

Tomczyk, S., Schou, J., Thompson, M.J.: 1995, Measurement of the rotation rate in the deep solar interior. Astrophys. J. Lett. 448, L57. DOI. ADS.

Torvalds, L.B.: 1991, Free Minix-like kernel sources for 386-AT. https://groups.google.com/d/msg/comp.os. minix/4995SivOI9o/GwqLJIPSICEJ.

Ulrich, R.K.: 1970, The five-minute oscillations on the solar surface. Astrophys. J. 162, 993. DOI. ADS.

Williams, H.K.: 2004, The construction of the Narrabri dome - 1992 August. BiSON Technical Report Series 227, High-Resolution Optical-Spectroscopy Group, University of Birmingham, UK. http://epapers. bham.ac.uk/2018/.

Willson, R.C.: 1979, Active cavity radiometer type IV. Appl. Opt. 18, 179. DOI. ADS. 